\title{
A Strömgren view of the multiple populations in globular clusters
}

\author{
E. Carretta ${ }^{1}$, A. Bragaglia ${ }^{1}$, R. Gratton ${ }^{2}$, V. D'Orazi ${ }^{2}$, and S. Lucatello ${ }^{2}$
}

1 INAF - Osservatorio Astronomico di Bologna, via Ranzani 1, 40127 Bologna, Italy
e-mail: [eugenio. carretta; angela. bragaglia] @oabo. inaf. it
2 INAF - Osservatorio Astronomico di Padova, Vicolo dell'Osservatorio 5, 35122 Padova, Italy
e-mail: [raffaele.gratton; valentina.dorazi; sara. lucatello]@oapd.inaf.it

Received 3 May 2011 / Accepted 8 September 2011

\begin{abstract}
We discuss a variety of photometric indices assembled from the uvby Strömgren system. Our aim is to examine the pros and cons of the various indices to find the most suitable one(s) for studying the properties of multiple populations in the globular clusters (GCs) discovered by spectroscopy. We explore in particular the capabilities of indices like $m_{1}$ and $c_{y}$ at different metallicities. We define a new index $\delta_{4}=(u-v)-(b-y)$ to separate first and second stellar generations in GCs of any metal abundance, since it keeps the sensitivity to multiple stellar populations over the full metallicity range and at the same time minimizes the sensitivity to photometric errors. We detect clear differences in the red giant branches of the GCs examined, such as skewness or bi/multimodality in color distribution. We connect the photometric information with the spectroscopic results on the $\mathrm{O}$ and $\mathrm{Na}$ abundances we obtained in our survey of GCs. Finally, we compute the effects of different chemical composition on the Strömgren filters and indices using synthetic spectra.
\end{abstract}

Key words. stars: abundances - stars: atmospheres - stars: Population II - globular clusters: general

\section{Introduction}

Thanks to the advent of efficient multiobject spectrographs at $8 \mathrm{~m}$-class telescopes and consequent extensive surveys, in the past few years it has become clear that the presence of multiple stellar populations is ubiquitous in globular clusters (GCs) (see, e.g., Marino et al. 2008; Carretta et al. 2009a,b, 2010c,b, 2011; Johnson \& Pilachowski 2010). There are at least two generations of stars, the second one formed from the ejecta of a fraction of the first generation (the so-called polluters, see e.g., Gratton et al. 2001, 2004). This is a crucial piece of information on the early phases of GC evolution. It seems to be related to their intrinsic, specific formation mechanism (Carretta 2006) and may give constrains to theoretical models of formation of massive stellar clusters.

With some delay with respect to spectroscopy, photometry now also indicates the presence of different stellar populations (not necessarily of different ages) in a subsample of clusters (see e.g. Anderson 1998; Bedin et al. 2004). We discuss some of its potentialities in the present paper, including strong sensitivity to abundances of some crucial element (namely N) and availability of large samples. However, most of the "hard" evidence of multiple populations in GCs is still based on spectroscopic surveys, where the multiple populations show up through a distribution of stars along the $\mathrm{Na}-\mathrm{O}, \mathrm{Mg}-\mathrm{Al}$, and $\mathrm{C}-\mathrm{N}$ anti-correlations, which have been found so far in all GCs where abundances of these elements in a large enough number of stars have been measured. Typically, modern spectroscopic surveys devoted to these studies include at most about 100 stars per cluster (e.g. Carretta et al. 2009a, and references therein) with internal errors in abundance, derived from intermediate and high-resolution spectra, typically representing about $8-10 \%$ of the total variance.

While many general information about the early evolution of GCs can be obtained from such distributions (e.g. the number ratios between first and second generations and the total extent of the Na-O anticorrelation, see Carretta et al. 2009a; Carretta et al. 2010c), others require both larger samples and smaller internal errors. In particular, this is the case for the distinction between smooth continuous distributions and discrete ones. Such a distinction is crucial, because it provides a basic clue to the nature of the polluters, which might be either fast rotating massive stars (lifetime of a few $10^{6}$ yrs: Decressin et al. 2007) or the most massive among intermediate mass stars, undergoing hot bottom burning during their AGB phase (lifetime of a few $10^{7}$ yrs: Ventura et al. 2001). For instance, discrete distributions might only be compatible with the AGB polluters, as suggested by Renzini (2008).

Moreover, while multiple populations are an intrinsic property of any GC (so that this might be adopted as a definition of a genuine GC, see Carretta et al. 2010e), the existing statistics also show that each GC does show difference in the shape and extensions of the $\mathrm{Na}-\mathrm{O}$ and $\mathrm{Mg}-\mathrm{Al}$ anticorrelations. In other words, apart from a common nucleosynthetic pattern, each cluster is characterized by its particular history, whose features may be studied, in principle, with high-resolution spectroscopy of a very large number of stars.

Alternative to spectroscopy, and less time consuming, photometry can provide both large samples and smaller internal errors. The most suitable photometric systems should couple a high sensitivity to the abundance variations related to the multiple population phenomena, with a high enough efficiency and the possibility of being applied to wide-field photometry. Sensitivity is obtained by passbands including strong molecular bands of CN (bandheads at 3883 and $4216 \AA$ ) or NH (around $3450 \AA$ ) or $\mathrm{CH}$ (around $4300 \AA$ ), since there are no common-use filters that are sensitive to the (smaller) spectral variations caused e.g., by different $\mathrm{Na}$ abundances. Several photometric systems have been considered, including broad band (see e.g. Carretta et al. 2010d; 
Lardo et al. 2011), intermediate band (see Grundahl et al. 1999), and narrow band ones (Lee et al. 2009). These different methods have advantages and limitations: for instance, Sloan filters (Stoughton et al. 2002) are very efficient, and high-quality photometry can be obtained for faint sources. However, sensitivity to the abundance variations is limited. On the other hand, while narrow band photometry may have very high sensitivity, results are not well understood yet (see Lee et al. 2009; and Carretta et al. 2010e). In this paper we consider results from Strömgren photometry in more detail, extending the previous work by Grundahl et al. (1999) and Yong et al. (2008).

Strömgren photometry (Strömgren 1956, 1966) was originally devised for F-dwarfs and is applied mostly to the study of hot and intermediate spectral type ( $O$ to $G)$ main sequence stars. There is, however, a long series of studies, (see e.g. Grundahl et al. 1998, 1999, 2002; Anthony-Twarog \& Twarog 2000; Anthony-Twarog et al. 2007) showing that it can also be successfully used for stars on the red giant branch (RGB) and on the horizontal branch (HB). In particular, Anthony-Twarog \& Twarog (2000) briefly discussed the effects of the "abundance anomalies" on the photometry of RGB stars in GCs. Grundahl et al. (1998) presented an extended Strömgren photometry of NGC 6205 (=M 13) and noted how the RGB stars presented a wide spread in the $c_{1}$ index. They attributed it to the different chemical compositions (not different iron content) of stars, because the relation between $c_{1}$ and the strength of $\mathrm{CN}$ and $\mathrm{CH}$ bands had already been demonstrated (e.g. in M 22 by Anthony-Twarog et al. 1995). A direct measure of $N$ abundance was obtained by Yong et al. (2008) and used to calibrate a new index $c_{y}$ (see Sect. 2.1). A similar approach was followed, for instance, by Carretta et al. (2009a) to connect the $c_{y}$ index to stars of the first (Na-poor) and of the second (Na-rich) generation in NGC 6752.

Sbordone et al. (2011) have recently presented a theoretical study of the influence of non-standard composition (e.g., enhanced in $\mathrm{Na}$ and/or $\mathrm{He}$, and/or light elements like $\mathrm{CNO}$ ) on the stellar models and the resulting isochrones, having in mind the particular case of the multipopulations in GCs. They considered the cases of Johnson $U B V I$ and Strömgren $u v b y$ filters. We discuss later (Sect. 5.1) the similarities and differences between their completely theoretical work and our observational approach. However, we note immediately that one of the most important is that their computations were for a single metallicity ${ }^{1}$ value, near the average for the Galactic GC population, while we instead considered GCs spanning (almost) the entire metallicity range for Milky Way GCs, from about -2.25 to about -0.70 dex.

In this paper we systematically and deliberately try to determine whether photometric data, in particular in Strömgren filters, can be used to distinguish between stars of different stellar generations in GCs by looking at their RGBs. We see that the situation is rather complex and that there is a strong dependence on the evolutionary phase and the metallicity considered. For instance, the index $c_{y}$ is efficient in separating different populations in GCs, but only at intermediate and low metallicity, while it loses sensitivity for metal-rich clusters (at least for the lower RGB). This sensitivity might even change its sign, making the interpretation of observational data ambiguous. The $m_{1}$ index is instead a good indicator of multiple populations at high metallicity. Finally, we explore the various indices that are

\footnotetext{
1 Within this paper, the term metallicity is usually adopted as a synonym of $[\mathrm{Fe} / \mathrm{H}]$, with an overall abundance pattern characteristics of halo stars, that is, with an excess of $\alpha$-elements.
}

Table 1. List of GCs.

\begin{tabular}{lccccc}
\hline \hline GC & Other & {$[\mathrm{Fe} / \mathrm{H}]$} & $E(B-V)$ & $y_{0}\left(\mathrm{RGB}_{\text {bump }}\right)$ & Telescope \\
\hline NGC 104 & 47 Tuc & -0.76 & 0.04 & 14.40 & Danish \\
NGC 6838 & M 71 & -0.82 & 0.25 & 14.00 & NOT \\
NGC 362 & & -1.17 & 0.05 & 15.30 & Danish \\
NGC 1851 & & -1.18 & 0.02 & 16.10 & Danish \\
NGC 288 & & -1.32 & 0.03 & 15.35 & Danish \\
NGC 6752 & & -1.55 & 0.04 & 13.50 & Danish \\
NGC 6205 & M 13 & -1.58 & 0.02 & 14.75 & NOT \\
NGC 6397 & & -1.99 & 0.18 & 12.10 & Danish \\
NGC 6341 & M 92 & -2.25 & 0.02 & 14.60 & NOT \\
\hline
\end{tabular}

Notes. [Fe/H]: Carretta et al. (2009c) and Carretta et al. (in prep.) for NGC 362; $E(B-V)$ : Harris (1996); $y_{0}\left(\mathrm{RGB}_{\text {bump }}\right)$ : this work.

possible to define, and we select a new index $\delta_{4}$ that uses all the four Strömgren filters uvby. We show that it is almost independent of reddening and that it can be used to efficiently separate different stellar generations in GCs over a broad range in metal abundance.

The plan of the paper is as follows. In Sect. 2 we present the available data on which we base our analysis, and we discuss the separation of GC stars in first and second generations as seen by the classical indices already known: $c_{y}$ and $m_{1}$. In Sect. 3 we examine other possibilities and present our new index $\delta_{4}$ and explore its sensitivity to metallicity and to the properties of different stellar generations. In Sect. 4 we study the correlations between $c_{y, 0}$ and $\delta_{4}$ and cluster parameters. In Sect. 5 we interpret our results with the aid of synthetic spectra. A summary is given in Sect. 6.

\section{The data}

We used the publicly available Strömgren photometry for nine Galactic GCs collected by Grundahl and coworkers (Grundahl et al. 1998, 1999, 2002) and presented by Calamida et al. (2007), who used them to obtain a new calibration of the metallicity index $m_{1}{ }^{2}$. These data were obtained at two different telescopes (Nordic Optical Telescope - NOT - on Canary Islands, Spain, and Danish telescope on La Silla, Chile), as indicated in Table 1. Instruments covering very different fields of view (about $4^{\prime}$ and $11^{\prime}$ on a side) were used; sometimes a single field was targeted, sometimes two or more. The observations generally comprise the GC center, with the exception of the two most metalrich cluster in the sample, NGC 104 (=47 Tuc) and NGC 6838 (=M 71). We refer to the Calamida et al. (2007) paper for references and details on the observations and calibrations.

We made a selection on photometric errors and sharpness (a parameter used to separate well measured stars from galaxies, defects, and cosmic rays), retaining only those stars with errors in all the four filters $\leq 0.02 \mathrm{mag}$ and $\mid$ sharpness $\mid \leq 0.2$. We used the 2MASS Point Source Catalog (Skrutskie et al. 2006) to astrometrize the catalogs through software written by P. Montegriffo at the Bologna Observatory. We employed the $E(B-V)$ values from Harris (1996) and corrected the $u, v, b, y$ magnitudes for reddening using the following relations $u_{0}=u-5.231 \times E(B-V), v_{0}=v-4.552 \times E(B-V)$, $b_{0}=b-4.049 \times E(B-V), y_{0}=y-3.277 \times E(B-V)$, with coefficients taken from Table 6 of Schlegel et al. (1998). From now on, all the quantities, colors, and combinations of bands are intended as dereddened. For seven of the clusters the reddening

\footnotetext{
2 We downloaded the catalogues from the web page http://www . oa-roma. inaf.it/spress/gclusters.html
} 
Table 2. Strömgren indices used in the paper.

\begin{tabular}{lccc}
\hline \hline Index & Definition & Alternative & Sensitive to \\
\hline$m_{1}$ & $(v-b)-(b-y)$ & $v-2 \times b+y$ & metallicity \\
$c_{1}$ & $(u-v)-(v-b)$ & $u-2 \times v+b$ & gravity \\
$c_{y}$ & $c_{1}-(b-y)$ & $u-2 \times v+y$ & gravity and $\mathrm{N}$ \\
$\delta_{4}$ & $(u-v)-(b-y)$ & $c_{1}+m_{1}$ & new index \\
$\delta_{4}^{\prime}$ & $\left(\delta_{4}-\delta_{4 B}\right) /\left(\delta_{4 R}-\delta_{4 B}\right)$ & & no $T_{\text {eff }}$ dependence \\
$\delta_{4}^{\prime \prime}$ & $\delta_{4}^{\prime} \times\left(\delta_{4 R, b}-\delta_{4 B, b}\right)$ & & \\
\hline
\end{tabular}

Notes. $\delta_{4 B}$ and $\delta_{4 R}$ are the $\delta_{4}$ values derived using the blue and red polynomial at the $y_{0}$ of each star (see text and Fig. 6). $\delta_{4 B, b}$ and $\delta_{4 R, b}$ are the same, but derived at the magnitude of the bump.

is very low $(E(B-V)=0.02$ to 0.05$)$. It is slightly higher for NGC 6397, but only for NGC 6838 some differential reddening is present. Finally, we estimated the magnitude of the RGB bump by visually inspecting the color-magnitude diagrams (CMDs). No sophisticated technique was applied, since this information was only used to roughly guide our selections (see below).

\section{1. $P, I$, and $E$ stars in Strömgren photometry, and $c_{y, 0}$}

For seven of the clusters we obtained FLAMES spectra of RGB stars in our ongoing survey to study the Na-O anticorrelation in a large sample of GCs with different parameters (see Carretta et al. 2007 for NGC 6752; Carretta et al. 2009a,b for NGC 104 (47 Tuc), NGC 6838 (M 71), NGC 288, and NGC 6397; Carretta et al. 2010b, 2011 for NGC 1851; Carretta et al. (in prep.) for NGC 362). We counteridentified stars in our spectroscopic samples, containing 50-150 objects each, and in the Strömgren catalogs. We generally found about 100 or more stars in common, except for NGC 104 and NGC 6838, where there are about 15 . The last two cases occur because our FLAMES fiber configurations are positioned around the cluster center, whereas the photometric observations are located off the cluster center. We used these stars to try to understand whether it is possible to differentiate stars of first and second generations (primordial - P - and intermediate plus extreme - I plus E respectively, in our notation: see Carretta et al. 2009a for the exact definition) on the basis of their position in color-magnitude diagrams (CMDs), experimenting with several combination of filters. All stars analyzed spectroscopically are members of the clusters, based on their derived radial velocities and chemical abundances.

For NGC 6205 (=M 13) and NGC 6341 (=M 92), we made a similar identification using literature papers. For NGC 6205, a cluster presenting an extended $\mathrm{Na}-\mathrm{O}$ anticorrelation (Sneden et al. 2004; Cohen \& Meléndez 2005), we used the measures of $\mathrm{Na}$ in Johnson et al. (2005) for the stars in common with our photometry ( 22 in total, two of which seem AGB, not RGB stars) to distinguish $\mathrm{P}$ and IE stars, since this paper includes more stars than the two cited above. For M 92 there are no extensive studies of the Na-O anticorrelations (Armosky et al. 1994 measured Na and $\mathrm{O}$ in only nine stars), but 34 stars have $\mathrm{Na}$ abundances in Sneden et al. (2000). We counter identified objects in Sneden et al. with the photometric data and found six stars in common (one of them is an HB star).

The $c_{y}$ index Yong et al. (2008) defined the index $c_{y}$ (see Table 2 for a summary of all the usual and new Strömgren indices used in the present paper) in their paper on NGC 6752; it traces the $\mathrm{N}$ abundance and was introduced because it is insensitive to the temperature, at first order, as shown from the near-verticality of the RGB at least up to the level of the RGB bump (see Fig. 1). The dependence of $c_{y}$ on $\mathrm{N}$ abundance comes from its definition, since it contains both the $u$ filter, where the strongest NH bands are present (at $\sim 3450 \AA$ ) and the $v$ one, where the $\mathrm{CN}$ features are present. In the latter they are weaker but the filter is weighted twice in this index. Given the definition of $c_{y}$, we might then expect sensitivity to $\mathrm{N}$ abundance but also a complex behavior. In fact, at low metal abundances and high temperatures, only the $\mathrm{NH}$ contribution should be important, because $\mathrm{CN}$ is negligible owing to the combination of the quadratic dependence on metal abundances ( $\mathrm{N}$ and C) and the strongest sensitivity to temperature of its formation. However, at high metallicity, absorption due to $\mathrm{CN}$ is much more relevant and may dampen, or even change, the sign of the sensitivity of $c_{y}$ on $\mathrm{N}$ abundances. More details are given in Sect. 5.

Figure 1 shows the $y_{0}, c_{y, 0}$ CMDs for the nine GCs, seven of which are in the FLAMES spectroscopic survey. As evident also from the enlargement of the RGBs presented in Fig. 2 (the lines are described in Sect. 4), the structure of the RGBs is different in the various clusters: the distribution in $c_{y, 0}$ can be rather uniform and define a more or less tight sequence (as in NGC 104 (=47 Tuc) or NGC 6341 (=M 92)), present a wide dispersion (NGC 1851), or show indications of skewness NGC 6205 (=M 13) and bimodality (NGC 288, NGC 6752). There are two suspect AGB stars in NGC $6205(=\mathrm{M} 13)$ and one confirmed HB star in NGC 6341 (=M 92, note that the HB is redder than the RGB in these plots). In Fig. 1 we identify $\mathrm{P}$ and IE stars with different colors (blue for $\mathrm{P}$ and red for IE stars, and we maintain the color-coding throughout the paper). The P stars (i.e., $\mathrm{Na}$ and N-poor first generation stars) tend to lie on a tight sequence on the blue side of the RGB, while the I and E ones (i.e., $\mathrm{Na}$ and $\mathrm{N}$-rich stars of the second generation), hereafter considered all together, occupy a wider color range on the red side. This is most evident in NGC 288, NGC 1851, and NGC 6752, and also clear in NGC 362 and NGC 6397. For the last, we also show in Fig. 1 the results from Lind et al. $(2009,2011)$; the separation between Na-poor and Na-rich stars is perhaps cleaner, but is based on a smaller sample (about 30 stars). In Carretta et al. (2009a) we could only classify 16 objects for NGC 6397 as P, I, and E, because we conservatively required both $\mathrm{Na}$ and $\mathrm{O}$ abundances. However, to separate stars into $\mathrm{P}$ and IE populations, it is necessary to know only $\mathrm{Na}$, so that we have all our 100 stars available for comparison. Only a few spectroscopically studied stars are present for NGC 104 and NGC 6838, but the two populations do not separate very clearly.

Such a segregation in color has already been found by Marino et al. (2008) in M4 (using the Johnson $U$ filter); in NGC 6752 by Carretta et al. (2009a), where we identified the N-rich stars in Yong et al. (2008) with the Na-rich ones; and in NGC 6397 by Lind et al. (2011), using the Strömgren index $c_{y}$ (and tightening the connection between abundance variations of different elements). The same effect (and the same explanation) was explored by Lardo et al. (2011) in nine GCs using the Sloan $u$ filter.

Our first conclusion is therefore that $c_{y, 0}$ seems to efficiently separate sequences of first and second generation stars at intermediate-to-low metallicities (indicatively for $[\mathrm{Fe} / \mathrm{H}] \lesssim$ -1.3), but does not work well for metal-rich clusters like NGC 104 or NGC 6838, or even NGC 362. We see in Sect. 5 that the effects of chemical abundances are complex.

In NGC 1851, even if the two stellar populations, P and IE, are segregated in $c_{y, 0}$ along the RGB for $y_{0}>15.5$, the branch has a wider spread in Fig. 1 with respect to the other GCs. Recently, Carretta et al. (2010b) have presented the hypothesis 

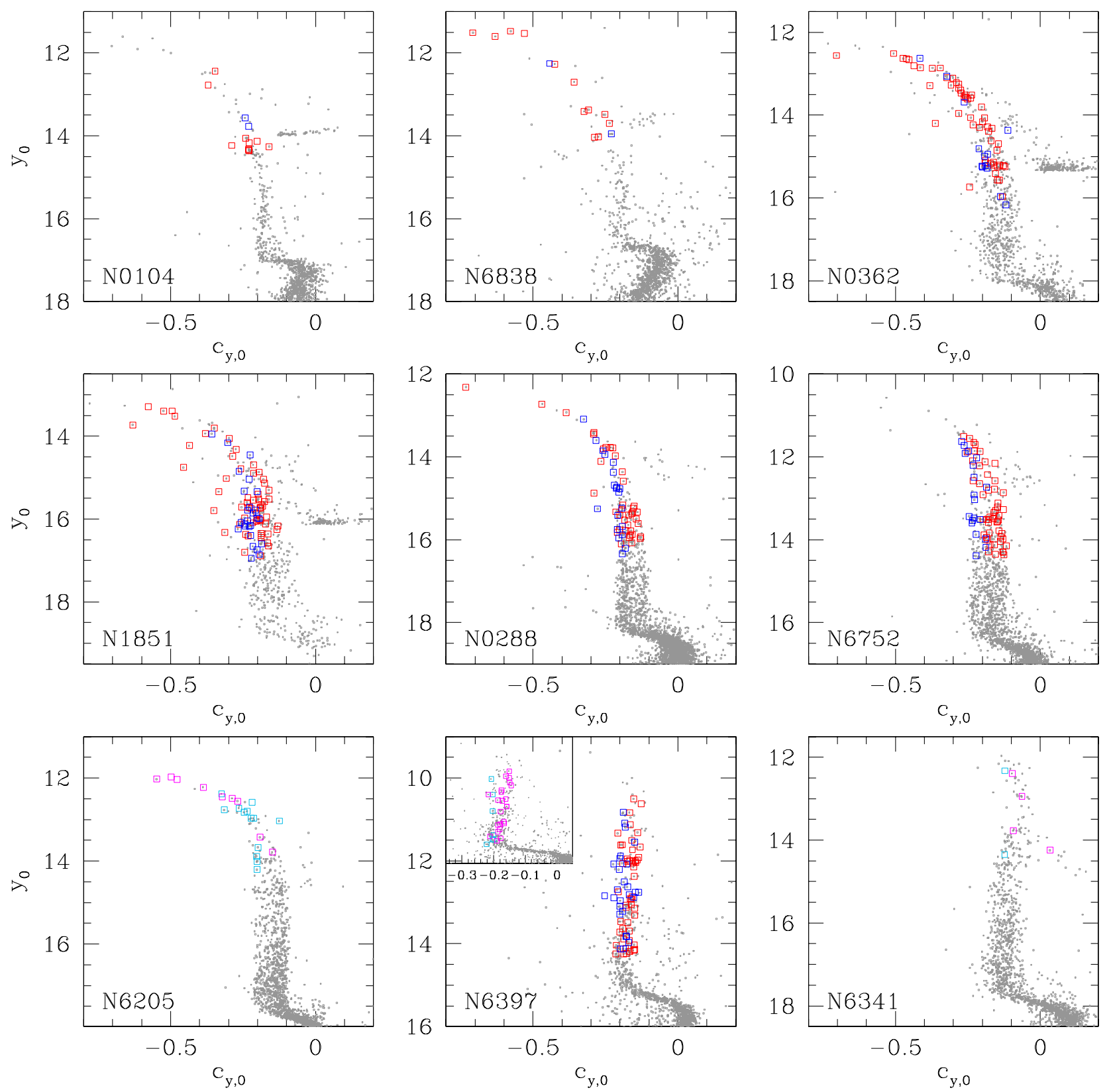

Fig. 1. CMDs in $y_{0}, c_{y, 0}$ for the nine GCs, seven in common with our spectroscopic survey. Blue symbols indicate P stars and red symbols IE stars, all confirmed members on the basis of their RVs. For NGC 6205 (=M 13) and NGC 6341 (=M 92), whose spectroscopic data are not homogeneous with the seven other GCs, we use light blue and magenta, respectively. For NGC 6397 we display in an inset the results from Lind et al. (2009, 2011), shown on our same scale in magnitude, and on an expanded scale in $c_{y, 0}$.

that NGC 1851 is the result of a merger of two originally distinct GCs with slightly different metallicity, each one with an Na$\mathrm{O}$ anticorrelation. The appearance of the plot would be explained if we are seeing the effect of a (narrow) spread in $[\mathrm{Fe} / \mathrm{H}]$ and likely overall CNO content (Yong \& Grundahl 2008), with Na$\mathrm{O}$ (hence $\mathrm{N}$ ) differences in both the metal-rich and metal-poor components. That $c_{y}$ measures both $\mathrm{N}$ and the overall metallicity is immediately visible using the template cluster with a dispersion in $[\mathrm{Fe} / \mathrm{H}]: \omega$ Cen. In Fig. 3 the most metal-poor population defines the right edge of the RGB, with stars of increasing metallicity populating sequences at more and more negative values of $c_{y}$. (We used the stars studied by Johnson \& Pilachowski 2010 with Strömgren photometry by Calamida et al. 2007.) The spectral synthesis calculations reported in Sect. 5 show that a variation in $[\mathrm{Fe} / \mathrm{H}]$ from -2.23 to -0.63 , roughly the range observed in $\omega$ Cen, causes changes in $c_{y}$ of $0.11 \mathrm{mag}$ for subgiants and $0.18 \mathrm{mag}$ for stars at the RGB bump, with $c_{y}$ smaller in more metal-rich stars. Such differences in $c_{y}$ are comparable to those expected between N-rich and N-poor stars. Similar differences are also produced by variations in the $\mathrm{CNO}$ content, within the limits that might be expected for stars in NGC 1851 and $\omega$ Cen (Yong \& Grundahl 2008; Marino et al. 2011). Untangling these effects can be difficult without further information.

\subsection{The $m_{1}$ index}

The Strömgren index usually employed to trace the metallicity is $m_{1}$. In Fig. 4 we display the effects of metallicity using the $m_{1}$ index, or better its dereddened version, $m_{0}$, displaying three representative monometallic clusters and NGC 1851. In NGC 104 
E. Carretta et al.: Strömgren photometry and MPs in GCs
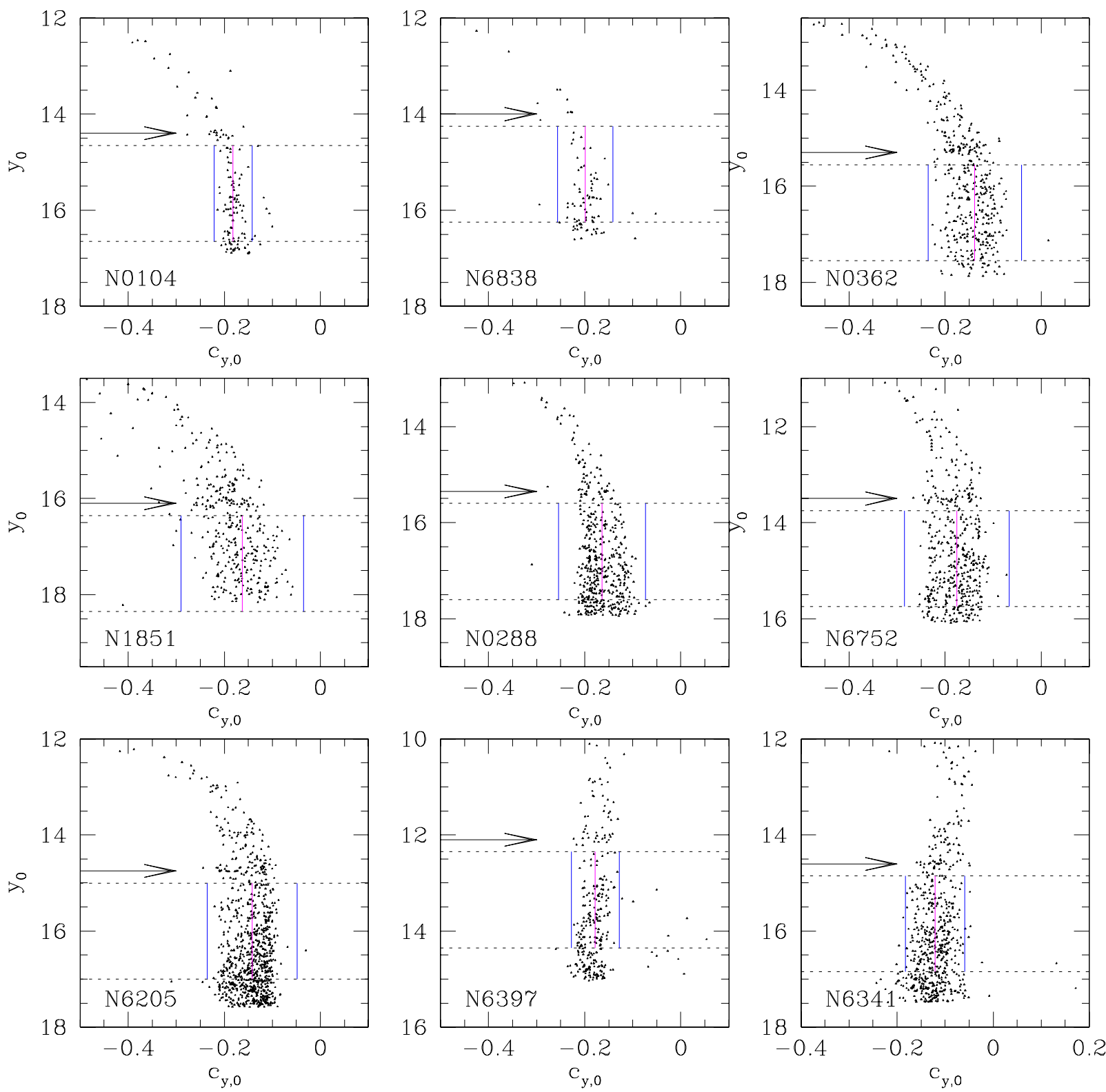

Fig. 2. Enlargement of the RGB region for the CMDs in $y_{0}, c_{y, 0}$ of the nine GCs. An arrow shows the assumed magnitude of the RGB bump. The lines indicate the RGB stars selected to derive average and rms values for $c_{y, 0}$ (see Sect. 4).

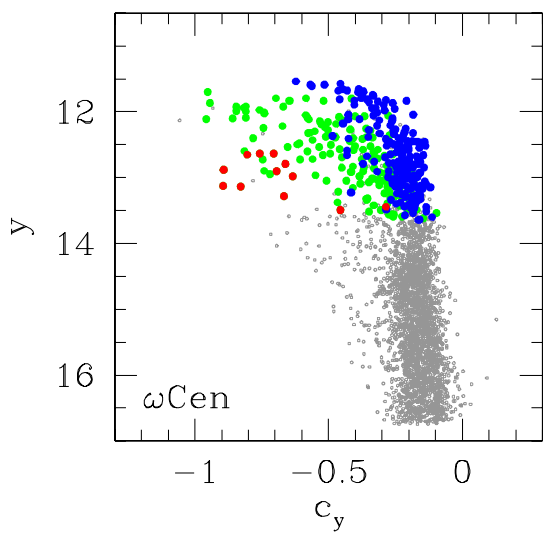

Fig. 3. CMD (not corrected for reddening) of $\omega$ Cen with stars of different $[\mathrm{Fe} / \mathrm{H}]$ (from Johnson \& Pilachowski 2010) indicated by different colors: blue stars have $[\mathrm{Fe} / \mathrm{H}]<-1.6$, red ones $>-1$, and green ones are in between.
(=47 Tuc) the RGB has a spread in $m_{0}$, but this cluster is well known to have a negligible dispersion in $[\mathrm{Fe} / \mathrm{H}]$ (e.g., Carretta et al. 2009c). Thus, this diagram indicates that the index $m_{0}$ is also sensitive to the abundance of elements other than iron at high metallicity (e.g., $\mathrm{N}$ is present through the $\mathrm{CN}$ bands visible in the $v$ filter, see discussion on $c_{y}$ above and Sect. 5). This dependence is almost not detectable for NGC 362, similar in metallicity to NGC 1851, and is undetectable in the intermediate metallicity NGC 6752 (where the apparent spread of the RGB fainter than $y_{0} \sim 13.5$ is due to the blue HB stars, which form an almost vertical sequence).

The $\mathrm{P}$ and IE stars occupy the bluer and redder parts of the RGB, respectively for NGC 104 (and NGC $6838=\mathrm{M} 71$, not shown here), while the separation is much less clear in NGC 362 and is not seen at all for NGC 6752 (and the other clusters of similar or lower metallicity). In NGC 1851 there is also a spread, not imputable to the blue HB or simply to a metallicity difference, since the dispersion in $[\mathrm{Fe} / \mathrm{H}]$ determined from 

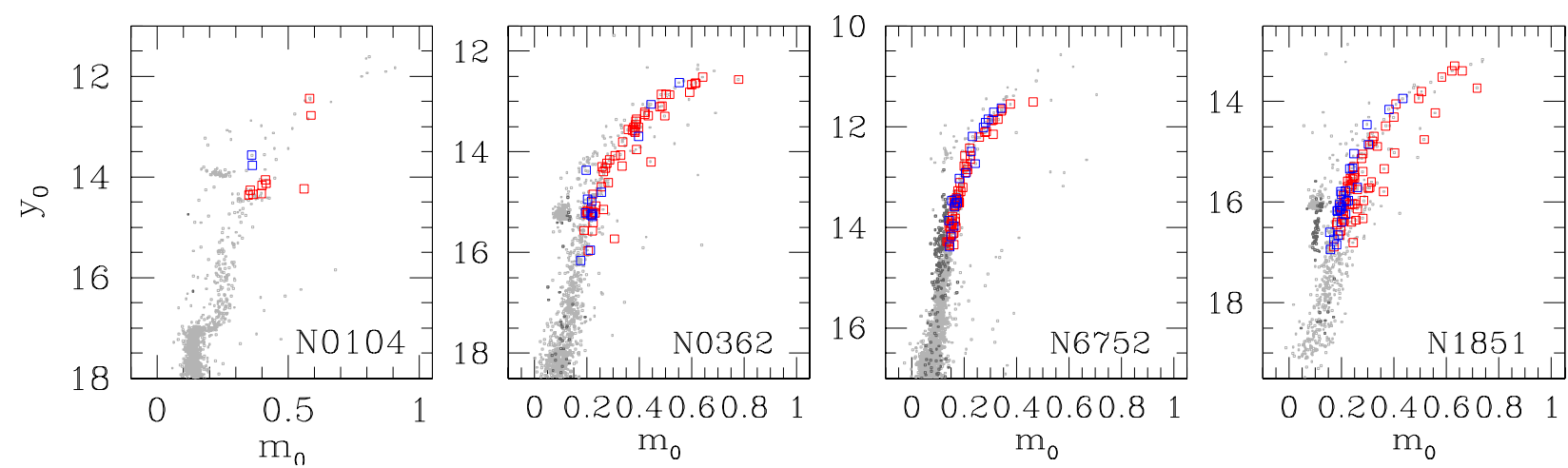

Fig. 4. CMDs in $m_{0}, y_{0}$ for three monometallic GCs and NGC 1851. Blue and red symbols have the same meaning as in Fig. 1; darker gray points, visible for NGC 6752 and NGC 1851, are blue HB stars (as indicated by their $b-y$ color).

spectroscopy should only yield a spread of $0.01 \mathrm{mag}$ in the $m_{1}$ index, according to our spectral synthesis (see Sect.5). This cluster is very complex, and many of its features are discussed in Carretta et al. (2011). Our second conclusion is then that $m_{0}$ is a decent indicator of first and second generations, but only at high metallicity (indicatively for $[\mathrm{Fe} / \mathrm{H}] \gtrsim-1.0$ ).

To summarize the results of this discussion, the index $c_{y}$ is a good indicator of different $\mathrm{N}$ abundances (i.e., distinct stellar generations) in metal-poor clusters. For metal-rich clusters, like NGC 104, the variations due to $\mathrm{N}$ in the $v-b$ and $u-v$ colors are similar and the net effect is to cancel or even to change the sign of the sensitivity of $c_{y}$ to different populations. In this metallicity regime $m_{1}$ is a better indicator.

\section{A new index, $\delta_{4}$}

To reduce any degeneracy with metallicity as much as possible, we test here a new Strömgren index, which we call $\delta_{4}$ and define as $\delta_{4}=(u-v)-(b-y)$, or $\delta_{4}=c_{1}+m_{1}$. We think that it can be an useful indicator for all metallicities and that it minimizes the dependence on photometric errors, since it does not weigh any of the photometric filters twice. Given the limit to photometric errors $<0.02$ mag adopted to select stars, the errors in $\delta_{4}$ should be $<0.08 \mathrm{mag}$. However, a more realistic estimate is an rms of $\sim 0.02 \mathrm{mag}$. Furthermore, both $m_{1}$ and especially $c_{y}$ do have a stronger weight on the bluest filters, the ones most probably having the largest photometric errors, especially for red stars. In our new index the photometric errors in $(u-v)$ and $(b-y)$ are not correlated with each other.

We have used only dereddened values in the present paper; however, $\delta_{4}$ would not depend much on reddening anyway. Taking from Strömgren (1966) the dependence on reddening for $c_{1}$ and $m_{1}$, we find that $E\left(\delta_{4}\right)=0.02 \times E(b-y)=0.014 \times$ $E(B-V)$, i.e., $\delta_{4}$ is approximatively reddening-free, at a firstorder approximation.

To test the ability of $\delta_{4}$ to separate stars of different generations, we plot in Fig. 5 the $\delta_{4}, y_{0}$ CMDs of the nine GCs, using the same symbols as in Fig. 1. The HB is redder than the RGB in these plots for intermediate and low metallicity and blue HB and the blue stragglers are almost horizontal sequences. The figure shows that there is a good separation between P and IE stars. Also using this new index we see some structure in the RGBs, most evident for two clusters, NGC 288 and NGC 6752; there the distribution of stars in $\delta_{4}$ seems bi-(multi)modal and not continuous. This is best shown by Fig. 6, where we only display the RGBs of all nine clusters on an enlarged scale. NGC 288 appears to have two distinct branches, one populated by $\mathrm{P}$, the other by IE stars (see the previous figure), while NGC 6752 displays a tri-modal distribution (see also Fig. 7). If we trust the link between $\delta_{4}$ and stellar generation, we could conclude that in NGC 288 there have been two episodes of star formation from gas that is homogenized well in each phase, without any appreciable continuous dilution from pristine gas, as generally invoked by models (e.g., Prantzos \& Charbonnel 2006) to explain the run of the observed Na-O anticorrelations. In NGC 6752 there are three populations, even if their separation is not as strikingly evident as in NGC 288.

The variation in $\delta_{4}$ seems to depend on magnitude, i.e. on temperature (Figs. 5, 6) and also on metallicity, see the next section. Ignoring this effect would produce a blurring of the $\delta_{4}$ values, and there would be the risk of potentially hiding some real features. Guided also by the distribution of $\mathrm{P}$ and IE stars that we see in Fig. 5, we traced reference sequences in the $\delta_{4}, y_{0}$ plane. First, we fitted a cubic polynomial in $y_{0}$ to the RGB ridge line; second, we labeled as "blue" the stars with negative residuals and "red" those with positive residuals with respect to it ( $\mathrm{P}$ and IE stars, respectively); and finally we drew the best-fitting cubic polynomial in $y_{0}$ to the two distributions of blue and red stars. The results are shown in Fig. 6 (where NGC 6752 is actually divided in three distributions).

These curves can help us eliminate the curvature of the RGB using both the blue and the red sequences, and immediately compare the distributions of red and blue populations. We then define a new index, called $\delta_{4}^{\prime}$ to rectify and normalize this index. Of course, with this procedure we risk normalizing to the errors in those cases where the intrinsic spread is narrow with respect to observational errors (as in the case of NGC 6397). We then defined a second index $\delta_{4}^{\prime \prime}$, which is $\delta_{4}^{\prime}$ multiplied by the rms scatter around the best-fit line for the whole sample (see Table 2 for definitions). It follows that $\delta_{4}^{\prime \prime}$ takes into account that the blue and red sequences can be more or less separated in color in different clusters. This index can be used to better identify the presence of multiple distributions along the RGBs. Given its definition, photometric errors on $\delta_{4}^{\prime \prime}$ should be similar to those in $\delta_{4}$, with typical rms values of $\sim 0.02 \mathrm{mag}$.

We also note that $\delta_{4}^{\prime}$ and $\delta_{4}^{\prime \prime}$ are only defined within a limited range in magnitude for each cluster, and extrapolation of the best-fit lines out of these regimes produce results that are clearly wrong. Our following discussion only considers those stars fainter than the RGB bump (or slightly brighter).

\section{1. $\delta_{4}$ and the separation of star generations}

Figure 7 shows the RGBs of the nine GCs "rectified" by using this new index, and brings out that strong differences do 

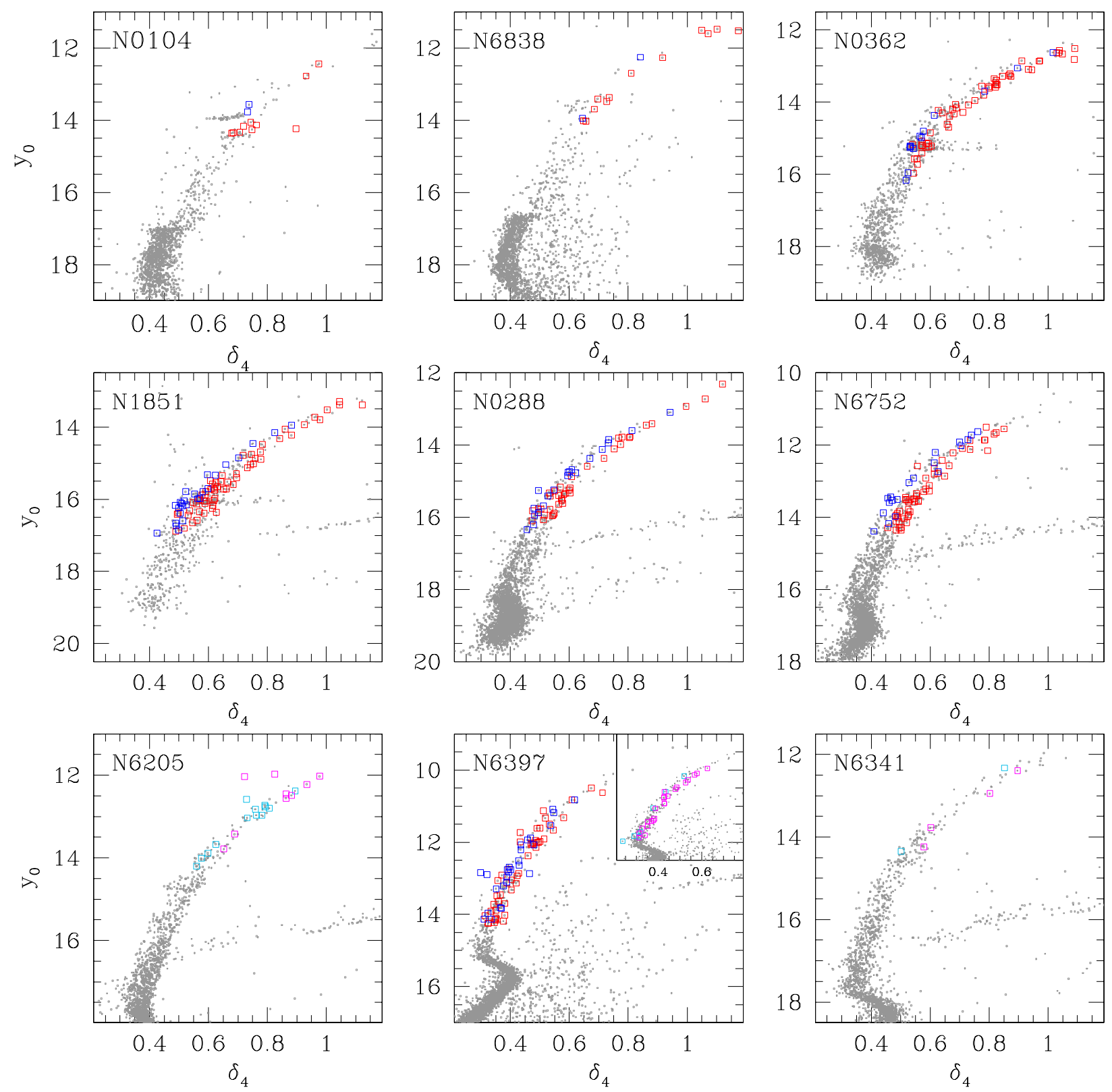

Fig. 5. CMDs in $\delta_{4}, y_{0}$ (see text for the definition of $\delta_{4}$ ). Blue symbols indicate P stars and red symbols IE stars (light blue and magenta for NGC 6205 (=M 13) and NGC 6341 (=M 92)). As in Fig. 1, for NGC 6397 we also show the results from Lind et al. (2009, 2011); the separation between Na-poor (light blue) and Na-rich (magenta) stars seems cleaner, due to the better data quality and the smaller sample.

exist among the GCs in our sample. This division is rather artificial in most cases: we simply divided second-generation (redder) and first-generation (bluer) stars by choosing the stars that lie near the red and blue lines in Fig. 6. The clear exceptions are NGC 288 and NGC 6752, since the distribution of giant stars is clearly bimodal in the first one, and trimodal in the latter. NGC 104 (=47 Tuc) and NGC 362 show smaller indications of separation, although a bimodal distribution is not excluded; NGC 6205 (=M 13) has perhaps the larger fraction of IE stars, and NGC 1851 has the largest dispersion in $\delta_{4}^{\prime \prime}$, possibly in agreement with the hypothesis of an origin by merger of two clusters with different chemical compositions (Carretta et al. 2010b, 2011).

To effectively see whether we can really discern a separation of populations, we also present the cumulative distribution in $\delta_{4}^{\prime \prime}$ in the same figure (limited to stars below the RGB bump). $\mathrm{A}$ change in the slope indicates separations into discrete distributions in colour (i.e., in population); NGC 288 and NGC 6752 are the most notable cases where such a feature is clearly present (and maybe NGC 104 and NGC 362).

Only in the most evident cases of NGC 288 and NGC 6752 can we estimate the fractions of second- ( red in the first, red plus green in the latter) and first-generation (blue) stars as defined by the photometry and the $\delta_{4}$ (and $\delta_{4}^{\prime \prime}$ ) index and compared them with the corresponding fractions of $\mathrm{P}$ and IE stars, defined spectroscopically. In Carretta et al. (2009a) we found that for all GCs the fraction of P stars is more or less constant, at a level of about one-third. Here we find the same result for NGC 6752 (blue stars $=27 \%$, red stars $=73 \%$, compared to $P=27 \%$ and IE $=73 \%$ ). However, for NGC 288 the fractions of P and IE stars are reversed $(62 \%$ and $38 \%$ from the photometry compared to $33 \%$ and $67 \%$ from the spectroscopy). The split in the RGB of NGC 288 is very clear (and is present, in the same sense, also in the $\left.c_{y, 0}, y_{0} \mathrm{CMD}\right)$. There are several things to be taken into 

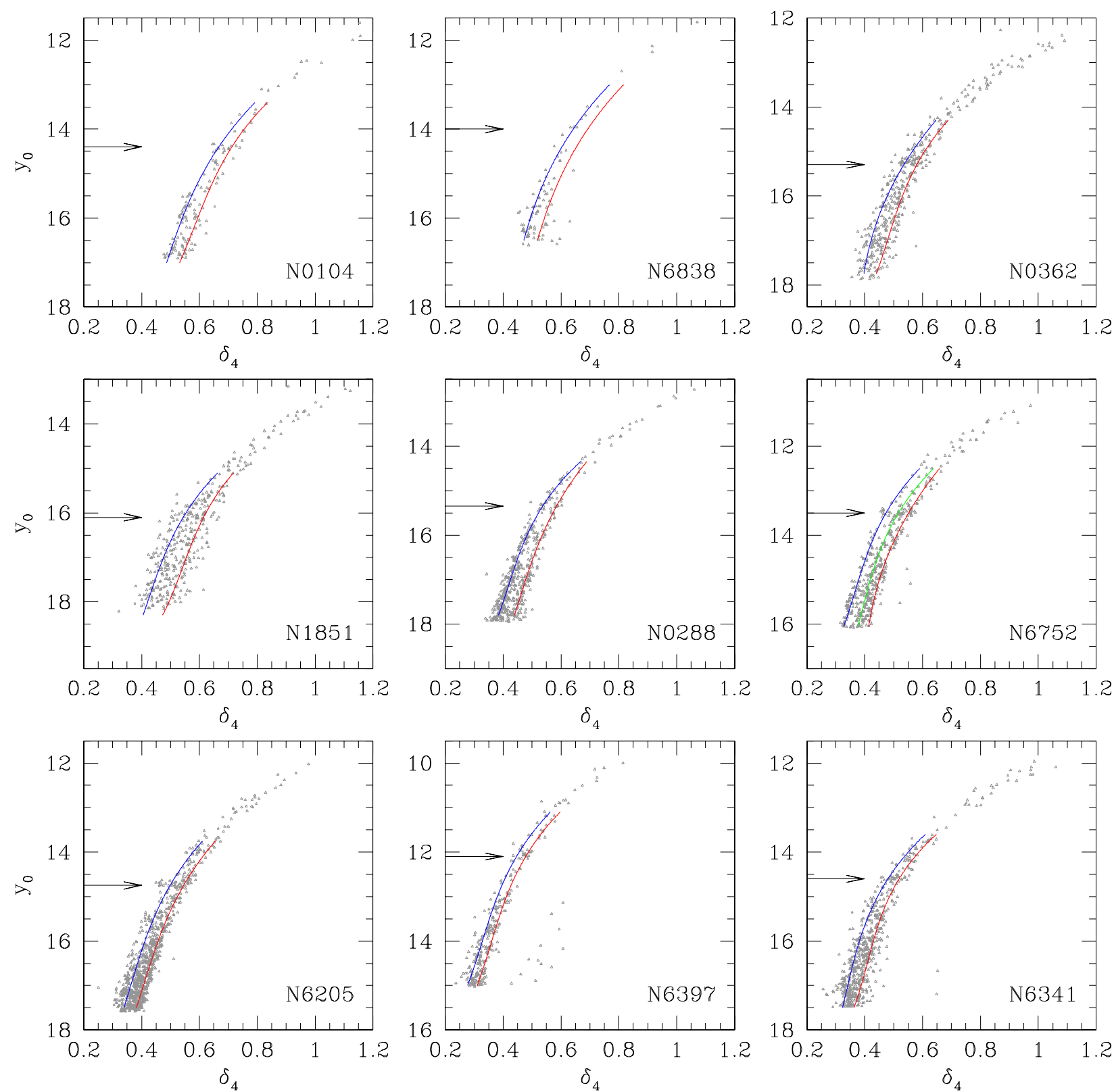

Fig. 6. CMDs in $\delta_{4}, y_{0}$ for the RGBs of the nine GCs, with the RGB bump level indicated by an arrow. The blue and red curves, stopped one magnitude brighter than the RGB bump, where the statistics begins to be too scarce, are polynomials describing the populations, drawn according to the distributions of P and IE stars in Fig. 5. For NGC 6752 the distribution of stars is trimodal (see text and next figure) and an intermediate (green) line is drawn.

account. First, the discrepancy is reduced if we classify stars in $\mathrm{P}$ and IE using only $\mathrm{Na}$ abundances, since in this case we have fractions of $42 \%$ and $58 \%$, respectively. Second, in NGC 288 the spectroscopic sample is limited, perhaps more than in other GCs, to stars that are significantly brighter than the bulk of the photometric sample, while the stronger concentration of blue/P stars is at the fainter limit of the RGB, where the actual separation is less evident. Third, we see in Figs. 1 and 5 that, at the fainter limits of the spectroscopic sample, there are a few IE stars that overlap the $\mathrm{P}$ ones in color. (The reverse does not happen.) In this particular cluster, the division in color/index is quite natural, while the spectroscopic classification in $\mathrm{P}$ and IE rests on the less defined minimum value for $\mathrm{Na}$ (see Carretta et al. 2009a, for a definition); indeed, a slightly different separation in Na would bring the fractions of $\mathrm{P}$ and IE stars into better agreement with the photometric ones. In conclusion, the result we found on the relative importance of first- and second-generation stars (one third and two thirds) is statistically valid for the whole sample of GCs, but in some clusters there may be individual variations. While this merits attention and further considerations, it is beyond the scope of the present paper.

Since the separation between populations mostly rests, spectroscopically, on the $\mathrm{Na}$ abundance, we plot in Fig. 8 the relation between $\delta_{4}^{\prime \prime}$ and the $\mathrm{Na}$ abundance, which is rather tight. Here we have divided stars in $\mathrm{P}$ and IE using only $\mathrm{Na}$ abundance, to increase the samples (we checked that this approach does not generally make a significant difference with respect to the criteria for PIE classification as adopted in Carretta et al. 2009a), and we also consider stars below the RGB bump since the separation between the sequences, hence the definition of $\delta_{4}^{\prime \prime}$, is better. We also show the case for NGC 6205 where, however, most of the observed stars are very bright. Nothing can be said for NGC 104 

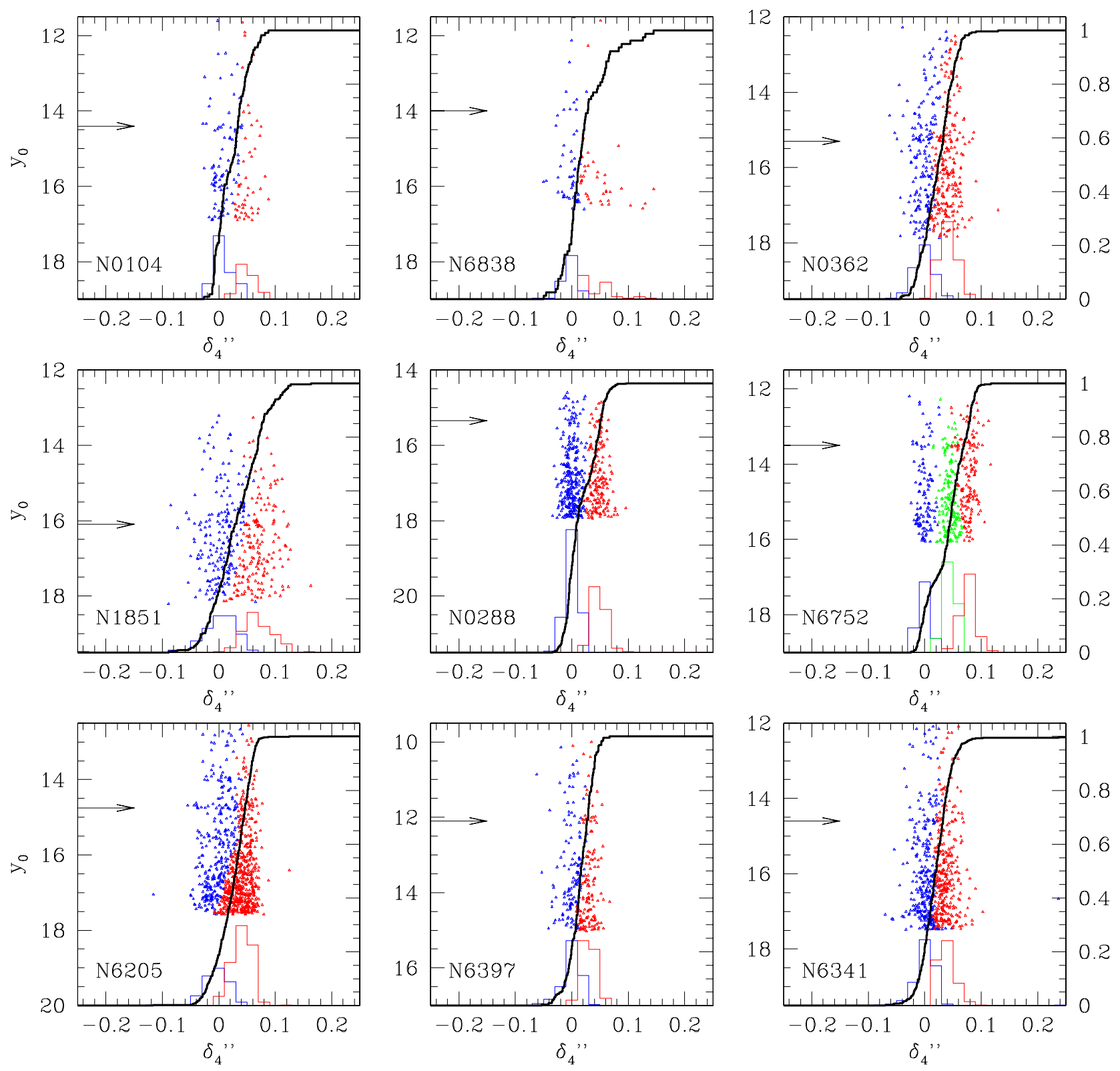

Fig. 7. Distribution in $\delta_{4}^{\prime \prime}$ for the nine clusters, showing the large difference among the GCs. The stars are colored in blue or red (and green, only for NGC 6752) according to the separation made in $\delta_{4}$ (see previous figure); this division is only indicative. The colored histograms refer to the blue/red populations. The black, heavy lines represent the cumulative functions (to which the left $y$-axes refer) of all stars below the RGB bump, indicated by an arrow.

and NGC 6838 (=M 71) since we have too few stars. For the other GCs, there is very good correlation between the $\delta_{4}^{\prime \prime}$ index and $\mathrm{Na}$, especially for the intermediate metallicity ones. (The sensitivity is worse at very low metallicity, like for NGC 6397 , where abundances are derived from rather weak lines.) The relations could be interpreted as continuous, but we see the possibility of separating stars into two groups, again most clearly in NGC 288 and NGC 6752, but also in NGC 1851 and especially in NGC 362 (where neither $\delta_{4}^{\prime \prime}$ or Na show well-defined partitions). It is sounder, and more natural, to separate the populations using both photometric and spectroscopic information, whenever possible.

\section{2. $\delta_{4}$ and metallicity}

Both $m_{1}$ and $c_{y}$ have a (complex) dependence on metallicity ([Fe/H] and $N$ abundance). To explore he dependence of $\delta_{4}$, we measured the value of $\delta_{4}$ at a reference magnitude $\left(M_{V} \simeq M_{y}=\right.$ 0 , i.e., near the level of the HB) and for the different populations, computing three $\delta_{4}$ values (blue, average, and red), guided by the natural separations and the lines drawn in Fig. 6. Given the rather good correlation existing between $\delta_{4}^{\prime \prime}$ index and $\mathrm{Na}$ abundances, we label the blue and red lines as $\mathrm{P}$ and IE respectively, even though it should be clear that the definition of PIE groups should be based on spectroscopy rather than on photometric data.

The values we obtain by this procedure for the clusters we examined are plotted in Fig. 9. We see that $\delta_{4}$ has a strong dependence on metallicity. The whole range of $\delta_{4}$ observed in NGC 6752 can be obtained if the metallicity varies by about 0.3 dex, which is, for instance, the difference between NGC 6752 and NGC 288. We then expect some difficulties in separating $\mathrm{N}$-rich and $\mathrm{N}$-poor populations in clusters like NGC 1851, where 

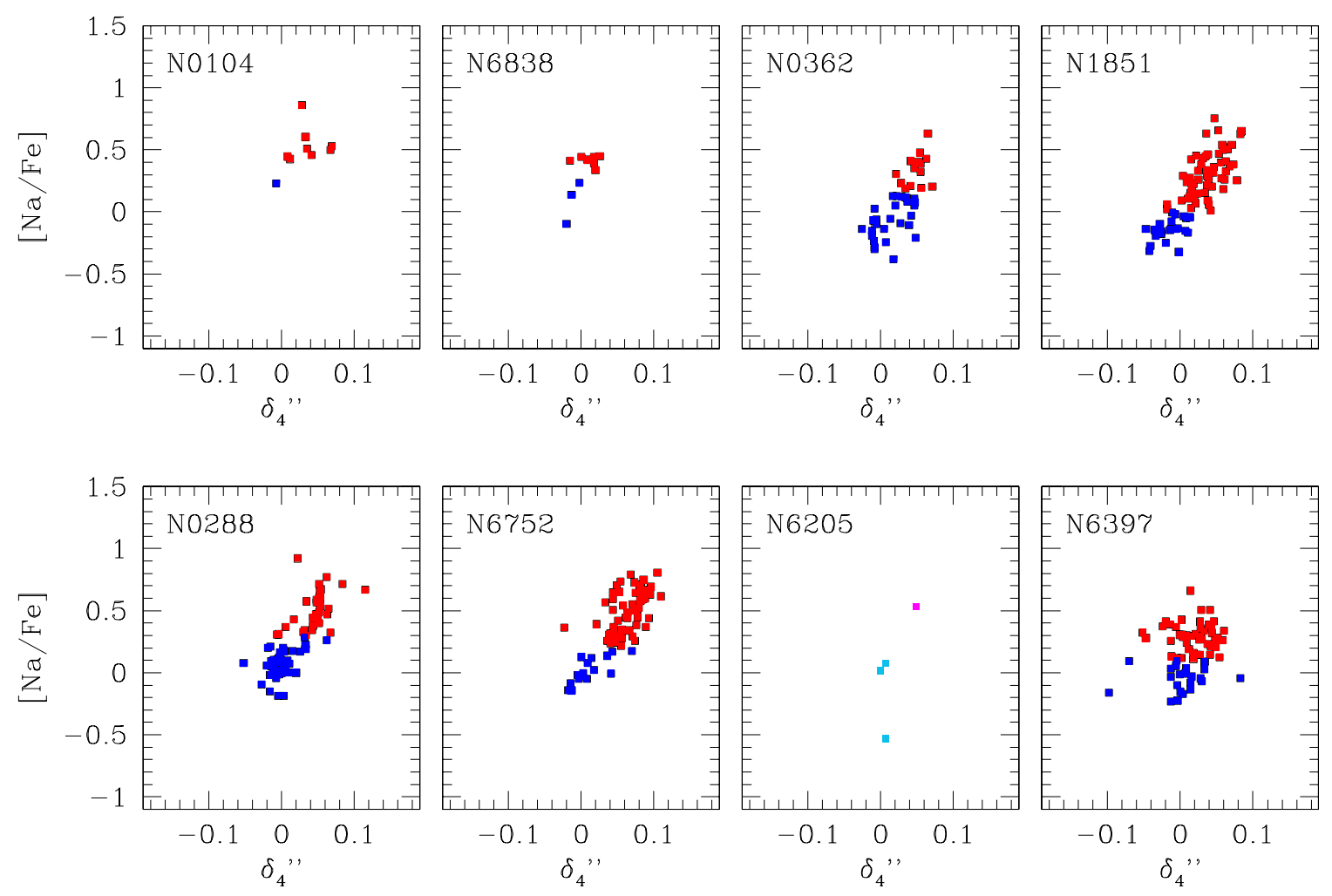

Fig. 8. Relation between $\delta_{4}^{\prime \prime}$ and the Na abundance for the seven GCs in our spectroscopic survey (and NGC 6205 = M 13, shown with different colors, as in Fig. 1). Blue and red symbols are for P and IE stars, respectively. (The definition is based only on Na abundance, to increase the sample sizes.)

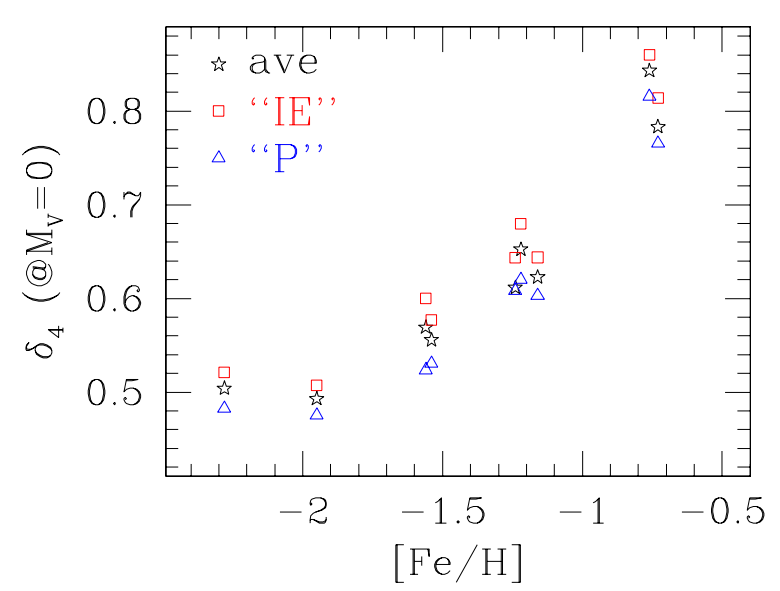

Fig. 9. Relation between metallicity and the value of $\delta_{4}$ at the reference magnitude $M_{\mathrm{V}}=0$ (i.e., at about the HB level) computed for the whole $\mathrm{RGB}$ and for the blue and red stars (P and IE, respectively).

there is an internal spread in metallicity. For it, Yong \& Grundahl (2008) and Carretta et al. (2010b) find a scatter of about 0.08 and 0.07 dex, respectively, well above the expected uncertainties. We conclude that $\delta_{4}$ is a very good estimator of the $\mathrm{N}$ abundance if a cluster is monometallic, as almost all are, at least at the precision we can reach with the present day spectra and analysis methods. (In Carretta et al. 2009c we showed, using UVES spectra of 19 GCs, that the internal homogeneity is at about $10 \%$, given an upper limit to the scatter of iron of less than 0.05 dex.) Where this is not valid, as in NGC 1851, it is better to separate the dependence from $[\mathrm{Fe} / \mathrm{H}]$ and $\mathrm{N}$ using, e.g., $m_{1}$. Of course, $\delta_{4}$ also has a dependence on temperature, as seen from the curvature of the RGBs.

\section{4. $c_{y, 0}, \delta_{4}$, and cluster parameters}

We have explored the possible relations of the $c_{y, 0}$ and $\delta_{4}$ indices with GC parameters. For $c_{y, 0}$, we concentrated on the stars below the RGB bump where the distribution in $c_{y, 0}$ is almost vertical, i.e. independent of the temperature (Fig. 2). As already discussed, the distribution in $c_{y, 0}$ is different from cluster to cluster and may present evidence of structures. Given the connection between this index and $\mathrm{N}$, these findings must be indicative of different distributions in N-richness among the GCs.

For each GC we only chose stars in a two-magnitude bin below the RGB bump (i.e., $y_{\text {bump }}+0.25$ to $y_{\text {bump }}+2.25$ ) and selected as RGB those falling within $3 \sigma$ from the average $c_{y, 0}$ value (after a $\kappa$-sigma clipping), with the selected region indicated in the figure. The adopted criteria allow us to deal with statistically significant samples of stars, and the verticality of the RGB in this plane allows us to separate the possible contamination by field stars rather easily. We measured the average value of $c_{y, 0}$ in the defined interval and its rms scatter. In principle, we expect that a higher average value does indicate a higher relative weight of the second generation, while a larger rms scatter could be the signature of a larger contribution of the nuclear processing by the first-generation polluters to the yield's budget in the cluster.

We show the best correlations we found for the average $c_{y, 0}$ and its rms in Fig. 10. As expected and also already visible in Fig. 2, the average depends on metallicity (left-hand panel in the second row of Fig. 10). We have tried to see if there are other significant correlations with several structural or chemical properties, but results are not very robust. The only highly significant correlations (significance of the Pearson coefficient greater than $99 \%$ ) are with metallicity for the average value of $c_{y, 0}$, and with IQR([Na/O]), $M_{\min }$, and $\delta Y$ for its rms. The average increases somewhat with the cluster mass (represented by its 

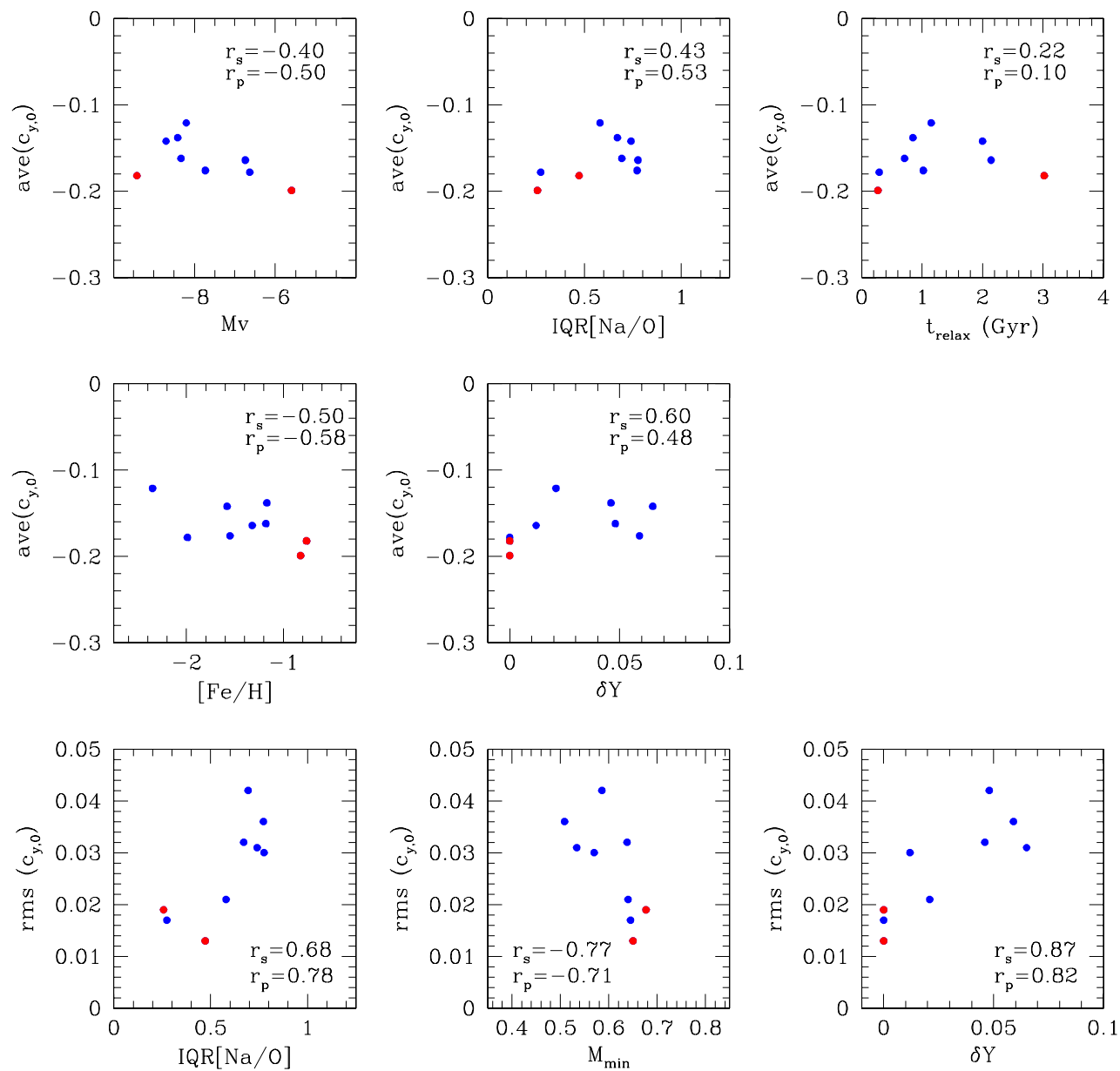

Fig. 10. Correlations of the average and rms for $c_{y, 0}$ with some interesting parameters. The two metal-rich clusters are indicated by red points. $r_{\mathrm{S}}$ and $r_{\mathrm{P}}$ are the Spearman's rank and Pearson's linear correlation coefficients, respectively.

proxy, the total absolute visual magnitude $M_{\mathrm{V}}$ ), and it is larger where the $\mathrm{Na}-\mathrm{O}$ anticorrelation is more extended (as indicated by the interquartile ratio IQR $([\mathrm{Na} / \mathrm{O}]$, see Carretta et al. 2009a). Similarly, it is related to $\delta \mathrm{Y}$, the amount of He-enhancement that can explain the long blue HB tails (Gratton et al. 2010) and which is connected with the extension of the Na-O anticorrelation. Some correlation exists with the relaxation time at halfmass radius, once 47 Tuc (which has the longest relaxation time in the sample) is excluded (rightmost, upper panel of Fig. 10). A full cluster relaxation occurs in at least two-three relaxation times; given the age of 47 Tuc (see, e.g., Gratton et al. 2010), this GC should be fully relaxed.

We note that 47 Tuc was already indicated by Carretta et al. (2010e) and Gratton et al. (2010) to be a peculiar cluster, with rather low values of IQR[Na/O] and He spread with respect to other clusters of similar total mass. The same is apparently visible in the correlation of the average $c_{y, 0}$ index and $M_{\mathrm{V}}$ in the present data. Recently, Lane et al. (2010) have suggested that this cluster might also be the result of a past merger of two originally distinct clusters. However, we must point out that for 47 Tuc there are fewer stars than in the other GCs, given the more external region sampled by the available Strömgren photometry. It is possible that at these larger distances from the cluster center, the ratio first-to-second generation stars is shifted toward the primordial population, as expected on theoretical grounds (e.g., D'Ercole et al. 2008) and as seen, e.g., from the distribution of CN-rich stars (Norris \& Freeman 1979), interpreted as Na-rich, second-generation ones in the present-day framework.
The last row of panels in Fig. 10 shows the best correlations we found using the dispersion in the $c_{y, 0}$ index. In the case of the rms scatter, the correlations with the spread of $\mathrm{He} \delta \mathrm{Y}$ (from Gratton et al. 2010) and IQR([Na/O]) are significant at more than $99 \%$. The first is mirrored by the anticorrelation between the spread in $c_{y, 0}$ and the $M_{\min }$, the minimum mass reached along the HB (also from Gratton et al. 2010). These findings are another indication that $c_{y}$ is tied to the different generations through its sensitivity to $\mathrm{N}$ (and the link with $\mathrm{Na}, \mathrm{O}$, and $\mathrm{He}$ abundances).

A similar approach can be taken for $\delta_{4}$, and we show the results in Fig. 11. Also in this case we used stars below the RGB bump and determined the average and rms (we used here the median, given the less uniform distribution in this index) of $\delta_{4}^{\prime \prime}$ for the nine clusters. The indications are similar to the previous case for $M_{\mathrm{V}}, \mathrm{IQR}, \delta Y$, and $M_{\min }$, while the correlation with the relaxation time disappears.

\section{Comparison of observed colors with synthetic spectra: interpretation}

To better understand the observed runs of the classical and new indices that we obtained from the Strömgren system, we computed several synthetic spectra over the wavelength region of interest (approximately, $\lambda=3000-6000 \AA$ ) for some cases of interest. The synthetic spectra were computed using the Kurucz (1993) set of model atmospheres (with the overshooting option switched off), and line lists from Kurucz (1993) CD-ROM's. 

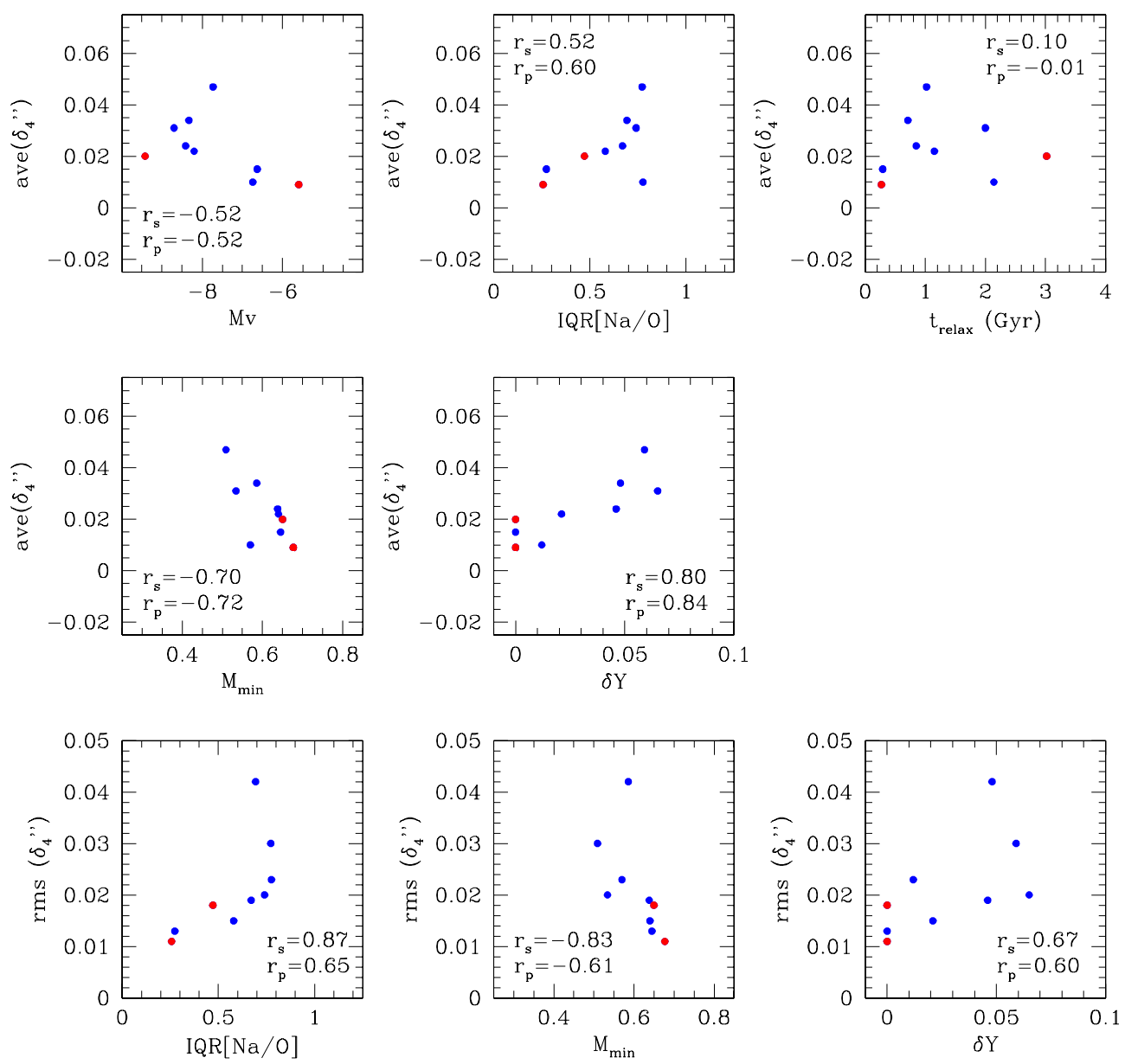

Fig. 11. Correlations of the median value and $\mathrm{rms}$ for $\delta_{4}$ with some interesting parameters. Symbols are as in previous figure.

It is well known that these line lists, while very extensive and useful, still have limitations. In addition, both the model atmospheres and our syntheses include approximations (e.g. monodimensional atmospheres, scattering treated as an additional absorption, etc.). For this reason we only consider differential effects between stars that should be very similar, except for details of their chemical composition.

All synthetic spectra were computed with a wavelength step of $0.02 \AA$. Then they were convolved with a Gaussian with an FWHM of $2 \AA$, and rebinned at about $1 \AA$ sampling for easier handling and display. The spectra were finally convolved with the transmission of the uvby (Strömgren 1956) and Ca filters (Anthony-Twarog et al. 1991), collected in the Asiago Database on Photometric Systems (Moro \& Munari 2000).

For all choices of atmospheric parameters, we considered as reference the spectra computed with the typical abundance pattern observed in field metal-poor stars, i.e., $[\mathrm{C} / \mathrm{Fe}]=[\mathrm{N} / \mathrm{Fe}]=0$, $[\mathrm{O} / \mathrm{Fe}]=[\mathrm{Mg} / \mathrm{Fe}]=[\mathrm{Ca} / \mathrm{Fe}]=0.4$, and $[\mathrm{Ti} / \mathrm{Fe}]=0.2$. Spectra computed with these parameters are labeled "N-poor". They should mimic the composition of $\mathrm{P}$ stars according to Carretta et al. (2009a). For the same choices of atmospheric parameters, we computed spectra for atmospheres having $[\mathrm{C} / \mathrm{Fe}]=-0.2$, $[\mathrm{N} / \mathrm{Fe}]=+1.3$, and $[\mathrm{O} / \mathrm{Fe}]=-0.1$ (and the same abundances for $\mathrm{Mg}, \mathrm{Ca}$, and $\mathrm{Ti}$ ). We labeled this second group of spectra "N-rich", and they should mimic the spectra of I stars ${ }^{3}$. In

\footnotetext{
3 We do not expect any significant change between I and $\mathrm{E}$ stars in this respect, insofar as the abundance of $\mathrm{O}$ is larger than that of $\mathrm{C}$. The main difference between I and $\mathrm{E}$ stars is in the $\mathrm{O}$ abundance, which changes
}

addition, we also explored the influence of $\mathrm{C}$ increase in a more limited way, by computing spectra for $[\mathrm{C} / \mathrm{Fe}]=0.2,[\mathrm{~N} / \mathrm{Fe}]=$ $0.0,[\mathrm{O} / \mathrm{Fe}]=0.4$ ("C-rich, N-poor" spectrum) and $[\mathrm{C} / \mathrm{Fe}]=0.2$, $[\mathrm{N} / \mathrm{Fe}]=1.3,[\mathrm{O} / \mathrm{Fe}]=-0.1$ ("C-rich,N-rich" spectrum). All these compositions are also indicated in Table 3.

First we considered pairs of "N-poor" and "N-rich" spectra for the four metallicities $[\mathrm{Fe} / \mathrm{H}]=-2.23,-1.63,-1.23$, and -0.63 . They have the effective temperature $T_{\text {eff }}$ and surface gravity $\log g$ appropriate for stars at the base of the subgiant branch (SGB), at the RGB bump, and at $M_{\mathrm{V}}=-1$ (as read from the isochrones of $\log ($ age $)=10.10$ by Bertelli et al. 2008). Table 3 lists the values of the atmospheric parameters we used and the offset in magnitude between the "N-poor" and "N-rich" spectra for the $u v b y$ and $C a$ bands and for various photometric indices $\left(m_{1}, c_{1}, c_{y}\right.$, and $\left.\delta_{4}\right)$.

Figure 12 presents an example for the synthetic spectra appropriate to stars at the RGB bump (panel a) and above the RGB bump (panel b) for $[\mathrm{Fe} / \mathrm{H}]=-1.23$, and also shows the transmission of the filters. This figure visually demonstrates what has already been discussed in the previous sections. The differences between the "N-poor" and "N-rich" spectra are

from $[\mathrm{O} / \mathrm{Fe}]$ about -0.1 to about -1 , but even in this case spectroscopic observations do not indicate the presence of very strong $\mathrm{C}$-bands, which are expected as soon as the abundance of $\mathrm{C}$ exceeds that of $\mathrm{O}$. The filters we are considering do not contain $\mathrm{OH}$ bands (e.g., the strong ones near $2700 \AA$ ) but only N molecules. However, N is already about $90 \%$ of the whole sum of CNO elements in I stars, and the small increase expected between I and E stars (about 5\%) does not influence our computations. 
Table 3. Model sensitivity of Strömgren photometric indices.

\begin{tabular}{|c|c|c|c|c|c|c|c|c|c|c|c|c|c|}
\hline Phase & $T_{\text {eff }}$ & $\log g$ & {$[\mathrm{~A} / \mathrm{H}]$} & $\Delta u$ & $\Delta v$ & $\Delta b$ & $\Delta y$ & $\Delta \mathrm{Ca}$ II & $\Delta m_{1}$ & $\Delta c_{1}$ & $\Delta c_{y}$ & $\Delta \delta_{4}$ & C-normal, N-poor minus \\
\hline SGB & 5578 & 3.44 & -2.23 & -0.056 & 0.002 & 0.000 & 0.000 & 0.003 & 0.002 & -0.061 & -0.061 & -0.059 & C-normal, N-rich \\
\hline RGB-bump & 5018 & 2.00 & -2.23 & -0.082 & 0.003 & 0.001 & 0.000 & 0.003 & 0.001 & -0.088 & -0.089 & -0.086 & rmal, N-rich \\
\hline$M_{\mathrm{V}}=-1$ & 4857 & 1.60 & -2.23 & -0.087 & 0.001 & 0.000 & 0.000 & 0.003 & 0.001 & -0.090 & -0.090 & -0.089 & C-normal, N-rich \\
\hline$\ldots$ & $\cdots$. & $\ldots$ & $\ldots$. & -0.062 & -0.003 & 0.000 & 0.000 & 0.003 & -0.003 & -0.056 & -0.056 & -0.059 & (a) \\
\hline SGB & 5537 & 3.53 & -1.63 & -0.099 & 0.007 & 0.000 & 0.000 & -0.002 & -0.007 & -0.086 & -0.086 & -0.092 & , N-rich \\
\hline RGB-bump & 5001 & 2.19 & -1.63 & -0.144 & -0.043 & 0.000 & 0.000 & -0.005 & -0.043 & -0.059 & -0.059 & -0.102 & C-normal, N-rich \\
\hline$M_{\mathrm{V}}=-1$ & 4683 & 1.51 & -1.63 & -0.175 & -0.102 & -0.002 & 0.000 & -0.007 & -0.101 & 0.027 & 0.028 & -0.071 & C-normal, N-rich \\
\hline .... & .... & $\cdots$. & $\ldots$ & -0.096 & -0.043 & 0.000 & 0.000 & -0.005 & -0.043 & -0.009 & -0.009 & -0.052 & 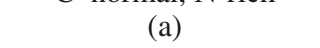 \\
\hline SGB & 5369 & 3.44 & -1.23 & -0.138 & -0.022 & 0.001 & 0.001 & 0.000 & -0.023 & -0.093 & -0.093 & -0.116 & $\mathrm{C}-\mathrm{n}$ \\
\hline RGB-bump & 4948 & 2.42 & -1.23 & -0.176 & -0.076 & 0.001 & 0.000 & -0.002 & -0.078 & -0.023 & -0.024 & -0.101 & C-normal, N-rich \\
\hline$\ldots$ & & $\ldots$ & $\cdots$ & -0.221 & -0.163 & -0.010 & -0.002 & -0.012 & -0.161 & 0.096 & 0.103 & -0.050 & C-rich, N-rich \\
\hline$M_{\mathrm{V}}=-1$ & 4478 & 1.40 & -1.23 & -0.189 & -0.149 & -0.002 & 0.000 & -0.006 & -0.145 & 0.107 & 0.109 & -0.038 & C-normal, N-rich \\
\hline$\ldots$. & $\ldots$. & $\ldots$. & $\ldots$. & -0.014 & -0.025 & -0.006 & -0.002 & -0.021 & -0.015 & 0.030 & 0.034 & 0.015 & C-rich, N-poor \\
\hline$\ldots$. & $\ldots$. & $\ldots$. & $\ldots$. & -0.251 & -0.274 & -0.027 & -0.006 & -0.021 & -0.226 & 0.270 & 0.291 & 0.044 & C-rich, N-rich \\
\hline .... & .... & ... & .... & -0.162 & -0.103 & -0.001 & 0.000 & -0.006 & -0.101 & 0.042 & 0.043 & -0.058 & (a) \\
\hline SGB & 5229 & 3.71 & -0.63 & -0.190 & 0.110 & -0.001 & 0.000 & -0.003 & -0.109 & 0.029 & 0.030 & -0.080 & $\mathrm{C}-1$ \\
\hline RGB- & 4765 & 2.53 & -0.63 & -0.211 & -0.180 & -0.003 & 0.000 & -0.007 & -0.175 & 0.146 & 0.149 & -0.029 & C-normal, N-rich \\
\hline$M_{\mathrm{V}}=-1$ & 4016 & 1.08 & -0.63 & -0.153 & -0.176 & -0.005 & -0.001 & -0.011 & -0.166 & 0.193 & 0.197 & 0.027 & C-normal, N-rich \\
\hline .... & $\ldots$. & $\ldots$. & $\ldots .$. & -0.097 & -0.123 & -0.002 & 0.000 & -0.011 & -0.118 & 0.146 & 0.148 & 0.028 & (a) \\
\hline
\end{tabular}

Notes. (a) $\Delta$ computed as C-normal, N-poor minus C-normal, N-rich, but with a composition changed after the RGB bump for C,N. C-normal, N-poor stars have: $[\mathrm{C} / \mathrm{Fe}]=[\mathrm{N} / \mathrm{Fe}]=0,[\mathrm{O} / \mathrm{Fe}]=[\mathrm{Mg} / \mathrm{Fe}]=[\mathrm{Ca} / \mathrm{Fe}]=+0.4,[\mathrm{Ti} / \mathrm{Fe}]=0.2 ; \mathrm{C}$-normal, N-rich stars have $[\mathrm{C} / \mathrm{Fe}]=-0.2,[\mathrm{~N} / \mathrm{Fe}]=$ $+1.3,[\mathrm{O} / \mathrm{Fe}]=-0.1$ (and the same abundances for $\mathrm{Mg}, \mathrm{Ca}$, and $\mathrm{Ti}$ ); $\mathrm{C}$-rich, $\mathrm{N}$-poor stars have $[\mathrm{C} / \mathrm{Fe}]=+0.2,[\mathrm{~N} / \mathrm{Fe}]=0.0, \mathrm{O}$ and other elements as in first case; $\mathrm{C}$-rich, $\mathrm{N}$-rich stars have $[\mathrm{C} / \mathrm{Fe}]=+0.2,[\mathrm{~N} / \mathrm{Fe}]=+1.3,[\mathrm{O} / \mathrm{Fe}]=-0.1$ and other elements as in the first case.
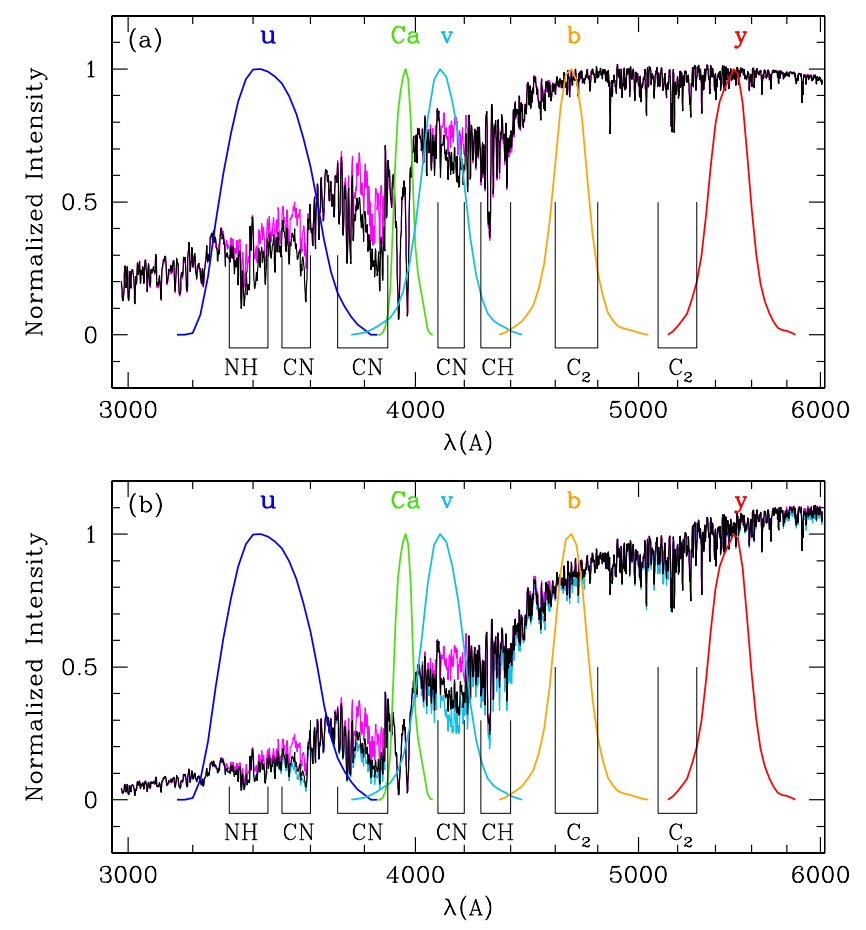

Fig. 12. a) Synthetic spectra for an N-poor (magenta), a N-rich (black), and a C-rich star at the RGB bump of a $\mathrm{GC}$ with $[\mathrm{Fe} / \mathrm{H}]=-1.23$ (see Sect. 4 for the various abundances used). Superimposed are the transmission curves for the $u v b y C a$ filters; some features of interest are indicated (see text). b) The same, but for a star above the RGB bump.

essentially due to the different strength of the molecular bands: the NH band at $\sim 3400 \AA$, which is stronger in the "N-rich" spectra and falls within the $u$ band; and the $\mathrm{CN}$ violet band at $\sim 4216 \AA$, which falls within the $v$ band. The $\mathrm{CH}$ A-X band at $\sim 4320 \AA$ is also different between the two spectra, but is near the edge of the $v$ band, so that its impact on the Strömgren colors is very weak. As a consequence, the flux predicted for both the $v$ and the $u$ bands is smaller for the "N-rich" spectra than for the reference "N-poor" ones. The difference is greater for the $u$ band, where it may be as much as $0.2 \mathrm{mag}$, save for the coolest and more metal-rich stars, where the effect is stronger in the $v$ band. This is due to both saturation and different temperature sensitivity of the molecular bands. A sketch of the variations in the $u, v, b, y, C a$ filters and in the three indices $m_{1}, c_{y}$, and $\delta_{4}$ is presented in Fig. 13 where we can appreciate their different sensitivities to $\mathrm{CNO}$ abundances along the various evolutionary phases and their run with metallicity. Table 3 and Fig. 13 can be used to interpret what we have seen in the observed CMDs in terms of different chemical compositions (in other words, of different stellar population).

While the largest variations between first- and secondgeneration stars are expected for $\mathrm{N}$, also the $\mathrm{C}$ variations can have an effect on the photometry. We computed only a couple of spectra, and Fig. 12 also shows the results for C-enhanced composition. The corresponding differences in the various filters/indices are indicated in Table 3. From these computations we see that, for $\mathrm{N}$-poor stars the effect of an increased $\mathrm{C}$ abundance is small, since the $\mathrm{CN}$ bands are not very strong and $\mathrm{O}$ is much more abundant than $\mathrm{C}$ in any case. In contrast, for $\mathrm{N}$-rich stars, an enhancement in $\mathrm{C}$ produces large changes to the Strömgren magnitudes, especially for the $u$ and $v$ filters. This means large differences in indices like $c_{y}$ and $m_{1}$ (while the differences almost cancel out for $\delta_{4}$, since it is the sum of the previous indices, whose variations have opposite signs).

The combination of $\mathrm{N}$ and $\mathrm{C}$ variations could then explain some peculiar cases, like NGC 1851, where we see cluster stars lying along an "anomalous", less populated RGB (see e.g. Fig. 4). In this cluster there have been (controversial) claims of 


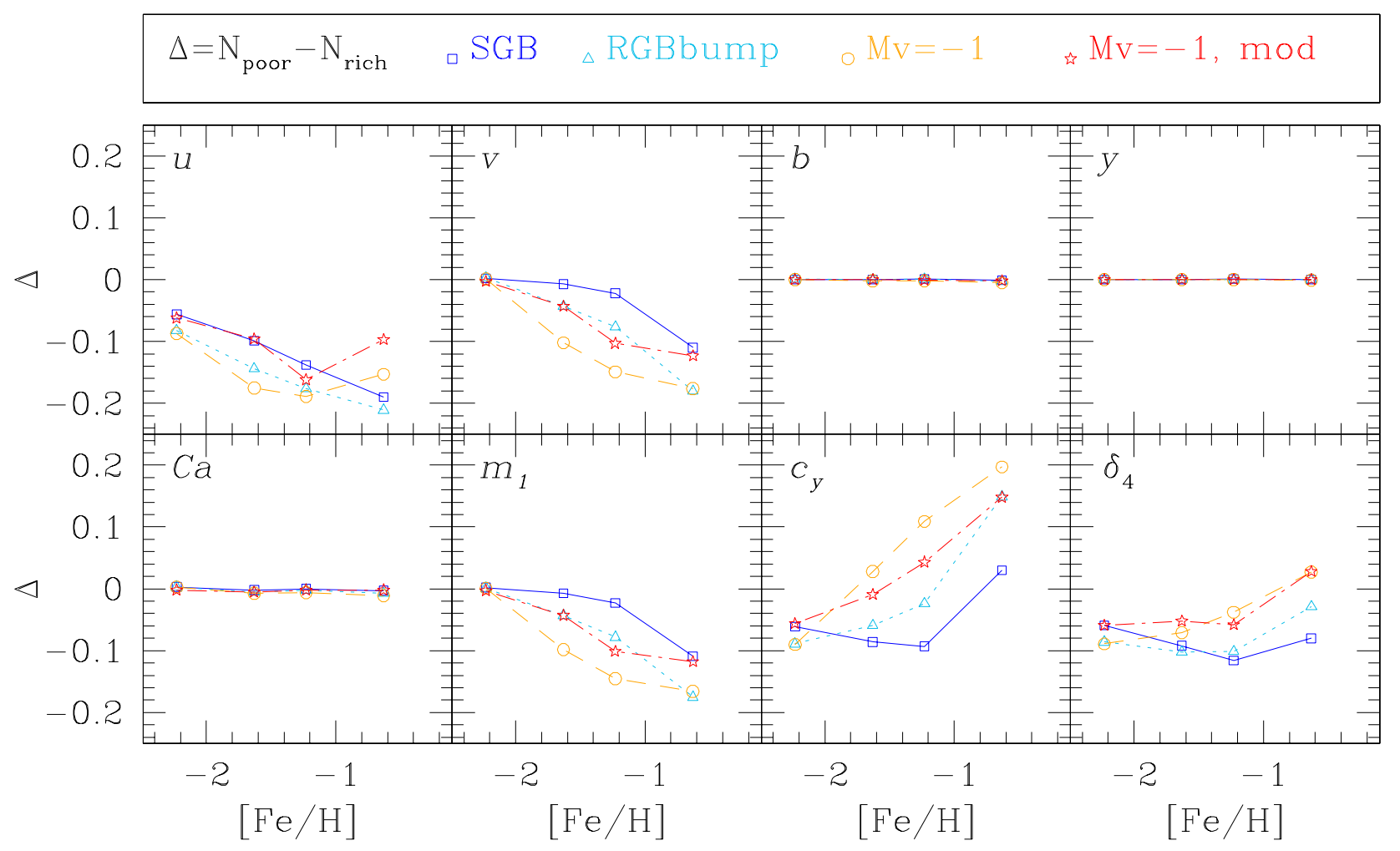

Fig. 13. Run with metallicity of the difference in magnitude and color for "N-rich" and "N-poor" stars (see Table 3). We show the three different evolutionary phases considered (two different compositions for the brighter star, see text). In each panel we indicate the filter/index involved.

variations in the sum of $\mathrm{C}+\mathrm{N}+\mathrm{O}$; this will be discussed in a paper in preparation. Here we simply propose that these stars could be the ones that are both $\mathrm{C}$ and $\mathrm{N}$-rich, while the ones with lower $\mathrm{N}$ abundance fall in with the bulk of the stars, even if C-rich.

Finally, we considered another possible composition difference. At the RGB bump there is a change in the surface abundance of $\mathrm{C}$ and $\mathrm{N}$ (see e.g., Gratton et al. 2000); i.e., C decreases and $\mathrm{N}$ increases, due to a mixing episode. In $\mathrm{C}$-normal, $\mathrm{N}$-poor stars the increase in $\mathrm{N}$ after the RGB bump almost compensates for the decrease in $\mathrm{C}$, and the $\mathrm{CN}$ bands do not change their strength. Instead, in $\mathrm{C}$-normal, $\mathrm{N}$-rich stars, the $\mathrm{N}$ abundance is already high and the $\mathrm{N}$ increase proportionally has lesser of an impact and does not compensate enough for the decrease in C. Hence, there is a smaller difference between an N-rich and an N-poor star if we take the evolutionary changes into account (see the correspondingly smaller differences in $u$ and $v$ and all correlated indices).

Armed with these results, we may then examine the prediction of our spectral synthesis on the observed indices.

i) $m_{1}=(v-b)-(b-y)$ is the classical metallicity index of the Strömgren photometry. In the present context, we notice that $b$ and $y$ (hence $b-y$, the classical temperature indicator) are insensitive to variations in the abundances of the CNO elements. For $m_{1}$, the only dependence is then contained in the $v$ magnitudes. Since the $\mathrm{CN}$ violet band is very weak in metal-poor and/or warm stars, $m_{1}$ only separates "N-poor" and "N-rich" for cool, metal-rich stars.

ii) $c_{1}=(u-v)-(v-b)$ is the classical gravity index of Strömgren photometry, because it measures the Balmer jump. In the present context, it essentially measures the difference between the strength of the $\mathrm{NH}$ and $\mathrm{CN}$ violet bands (the latter actually weighted twice). Therefore it efficiently separates "N-poor" and "N-rich" at low metallicities (where the $\mathrm{CN}$ violet band is negligible), but it changes the sign of its sensitivity for cool metal-rich stars, where the $\mathrm{CN}$ violet band is quite strong. The same holds for $c_{y}\left(=c_{1}-(b-y)\right)$, which has the same sensitivity on CNO abundances of $c_{1}$.

iii) Our new index $\delta_{4}=(u-v)-(b-y)$ keeps the sign of its sensitivity to $\mathrm{CNO}$ abundances constant over a range of temperature/metallicity/gravity that is wider than for $m_{1}$ and $c_{1}$ or $c_{y}$, although it also fails for the coolest and most metalrich stars. The reason for this more uniform behavior is that it weighs the $v$ band only once, and it is then less sensitive to the strength of the $\mathrm{CN}$ violet bands. In practice, the sensitivity of $\delta_{4}$ on CNO abundances is all contained in the $(u-v)$ term. The addition of the $(b-y)$ term is, however, useful for reducing its sensitivity to temperature and reddening. In particular, since $\delta_{4}$ is practically insensitive to reddening, it can also be safely used for clusters with strong differential reddening.

\subsection{Comparison with Sbordone et al. (2011)}

This very recent paper approaches the problem of the photometric signatures of multiple populations in GCs from the theoretical point of view. The approach and purposes were different, since the authors wanted to present full blown isochrones and take the effect of helium in consideration as well, while we concentrated on the RGB. They then computed self-consistent stellar models, taking the different chemical compositions of firstand second-generations stars into account both for the stellar interiors and the stellar atmospheres. They computed models with several compositions (standard mixture, NaCNO-enhanced, He-enhanced) and translated the theoretical isochrones into the observational planes for Johnson and Strömgren photometry, showing the effects on the main sequence, SGB, and RGB. 
While addressing the same problem, our paper and theirs differ in several points. (i) While we have worked on the observational data of several GCs, they did not present a direct comparison with observed cases, deferring this to subsequent papers. (ii) We did not compute new, self-consistent atmospheres and fluxes, but only the variation in the absorption spectrum due to a change in composition, using a standard model. Their approach is formally better; however, as shown also by their paper, there is little difference between the two approaches, since the molecular bands present in these stars affect only a small fraction of the total flux, and the various models differ only in the most external parts near the stellar surface. In fact, Gustafsson et al. (2008) consider the impact of mild and heavy $\mathrm{CN}$ cycling on the structure of the atmospheres and find that it is negligible for the temperatures of interest in our computations (see their Fig. 7). (iii) In our paper the changes in composition due to the first dredge-up and the extra-mixing after the RGB bump are taken into consideration. This has a significant impact on the derived results, since $\mathrm{C}$ and $\mathrm{N}$ are involved in these changes. (iv) Perhaps most important is that they do all calculations for only one value of metallicity, while we explore the entire range of metallicity of Galactic GCs. This is an important factor, since we have seen that there is a strong dependence on metallicity of the sensitivity of the various colors and indices to different compositions (see Table 3 and Fig. 13).

Given all these differences, it is legitimate to ask whether we are obtaining similar results. Taking our case at $[\mathrm{Fe} / \mathrm{H}]=-1.63$ to compare to their -1.62 and looking at their Fig. 8, we obtain similar, but smaller, differences between $\mathrm{N}$-rich and poor compositions in $u$ (and of course $b$ and $y$, where $\mathrm{N}$ has no influence). There are instead discrepancies in the $v$ filter, where we find differences and they do not. However, there is a large difference in $\mathrm{C}$ abundance between the two cases. We use $[\mathrm{C} / \mathrm{Fe}]=-0.2$, compared to their -0.6 , meaning that their model has $[\mathrm{C} / \mathrm{H}]=$ -2.23 , i.e., is more similar to our $[\mathrm{Fe} / \mathrm{H}]=-2.23$ case, where we too do not see any effect in the $v$ filter (the $\mathrm{CN}$ bands are really very weak in these models).

The large difference in $\mathrm{C}$ abundance and the single, quite low metallicity could also explain the fact that their models predict larger differences in $c_{y}$ between the different compositions (see their Fig. 15) than our computations (Table 3). In practice, we have stronger $\mathrm{CN}$ bands in our spectra and this has an effect on the $v$ filter, which is actually weighted twice in the definition of $c_{y}$, and outweighs the effect on the $u$ filter, where the NH band dominates. A more complete comparison between the two works is difficult and outside the goals of the present work. It could be interesting, however, to merge the two approaches, to better define the properties of multiple populations in GCs.

\section{Summary}

In this paper we explored the capabilities of the Strömgren photometric system to characterize multiple populations that we firmly know (from spectroscopy) to be harbored in all GCs. Thanks in particular to the $u$ filter, which encompasses the molecular NH band at $3400 \AA$, and the $v$ filter, at least for the metal-rich GCs, it is possible to separate N-poor (Na-poor, $\mathrm{O}$-rich, first generation) stars well from $\mathrm{N}$-rich (Na-rich, O-poor, second generation) stars using Strömgren multicolor photometry.

In our systematic exploration of the parameter space, using Grundahl's (and coworkers) photometry made available by Calamida et al. (2007) for nine GCs, we found that the classical metallicity index $m_{1}$ is able, in the present context, to separate only cool and metal-rich stars into $\mathrm{N}$-strength classes. Both the typical gravity indicator $c_{1}$ and its improved version $c_{y}$ defined by Yong et al. (2008), work well in separating N-rich and N-poor stars at low metallicity, but give ambiguous results in the metalrich and cool star regimes.

We defined a new indicator, $\delta_{4}$, that seems to works better than the previous indices over a wider range of metallicity, although even in this case the separation of different populations is not achieved well in the cool and metal-rich regime.

The variations that are sampled by all these indices are accounted for by changes in the abundances of CNO elements, as demonstrated by several comparisons with synthetic spectra convolved with the transmission filter. However, further work is necessary, combining for instance our observational approach with the theoretical one chosen by Sbordone et al. (2011).

In the present paper we have limited our analysis to the RGB stars to ensure the best photometric data and to compare them directly with the available firm constraints given by the chemical abundances (e.g., the Na-O anticorrelation). The next step will be extensive analysis of photometric data over wide fields also reaching extremely blue (faint) $\mathrm{HB}$ and main sequence stars with comparable precision. The gain in statistics will permit us to better study hot issues such as the radial distribution of multiple stellar populations in GCs, which is tightly connected with the formation and evolution of these stellar aggregates.

Acknowledgements. We wish to thank the referee Luca Sbordone for his careful report, definitely useful for improving the paper. We used data from the Two Micron All Sky Survey, which is a joint project of the University of Massachusetts and the Infrared Processing and Analysis Center/California Institute of Technology, funded by the National Aeronautics and Space Administration and the National Science Foundation. This research made use of the SIMBAD and ViZier databases, operated at the CDS, Strasbourg, France and of NASA's Astrophysical Data System. Financial support from PRIN-MIUR 2007 "Multiple Stellar Populations in Globular Clusters: Census, Characterization and Origin", and PRIN-INAF 2009 "Formation and Early Evolution of Massive Star Clusters", is acknowledged.

\section{References}

Anderson, J. 1998, Ph.D. Thesis

Anthony-Twarog, B. J., \& Twarog, B. A. 2000, AJ, 120, 3111

Anthony-Twarog, B. J., Twarog, B. A., Laird, J. B., \& Payne, D. 1991, AJ, 101, 1902

Anthony-Twarog, B. J., Twarog, B. A., \& Craig, J. 1995, PASP, 107, 32

Anthony-Twarog, B. J., Twarog, B. A., \& Mayer, L. 2007, AJ, 133, 1585

Armosky, B. J., Sneden, C., Langer, G. E., \& Kraft, R. P. 1994, AJ, 108, 1364

Bedin, L. R., Piotto, G., Anderson, J., et al. 2004, ApJ, 605, L125

Bertelli, G., Girardi, L., Marigo, P., \& Nasi, E. 2008, A\&A, 484, 815

Calamida, A., Bono, G., Stetson, P. B., et al. 2007, ApJ, 670, 400

Carretta, E. 2006, AJ, 131, 1766

Carretta, E., Bragaglia, A., Gratton R. G., Lucatello, S., \& Momany, Y. 2007, A\&A, 464, 927

Carretta, E., Bragaglia, A., Gratton, R. G., et al. 2009a, A\&A, 505, 117

Carretta, E., Bragaglia, A., Gratton, R.G., \& Lucatello, S. 2009b, A\&A, 505, 139

Carretta, E., Bragaglia, A., Gratton, R., D’Orazi, V., \& Lucatello, S. 2009c, A\&A, 508, 695

Carretta, E., Bragaglia, A., Gratton, R.G., et al. 2010a, ApJ, 712, L21

Carretta, E., Gratton, R. G., Lucatello, S., et al. 2010b, ApJ, 722, L1

Carretta, E., Bragaglia, A., Gratton, R. G., et al. 2010c, ApJ, 714, L7

Carretta, E., Bragaglia, A., D’Orazi, V., Lucatello, S., \& Gratton, R.G. 2010d, A\&A, 519, A71

Carretta, E., Bragaglia, A., Gratton, R.G., et al. 2010e, A\&A, 516, A55

Carretta, E., Lucatello, S., Gratton, R., Bragaglia, A., \& D’Orazi, V. 2011, A\&A, 533, A69

Cohen, J. G., \& Meléndez, J. 2005, AJ, 129, 303

Decressin, T., Meynet, G., Charbonnel, C., Prantzos, N., \& Ekstrom, S. 2007, A\&A, 464, 1029 
D'Ercole, A., Vesperini, E., D'Antona, F., McMillan, S. L. W., \& Recchi, S. 2008, MNRAS, 391, 825

Gratton, R. G., Sneden, C., Carretta, E., \& Bragaglia, A. 2000, A\&A, 354, 169

Gratton, R. G., Bonifacio, P., Bragaglia, A., et al. 2001, A\&A, 369, 87

Gratton, R., Sneden, C., \& Carretta, E. 2004, ARA\&A, 42, 385

Gratton, R. G., Carretta, E., Bragaglia, A., Lucatello, S., \& D’Orazi, V. 2010, A\&A, 517, A81

Grundahl, F., \& Briley, M. 2001, NuPhA, 688, 414

Grundahl, F., Vandenberg, D. A., \& Andersen, M. I. 1998, ApJ, 500, L179

Grundahl, F., Catelan, M., Landsman, W. B., Stetson, P. B., \& Andersen, M. I. 1999, ApJ, 524, 242

Grundahl, F., Stetson, P. B., \& Andersen, M. I. 2002, 395, 481

Gustafsson, B., Edvardsson, B., Eriksson, K., et al. 2008, A\&A, 486, 951

Harris, W. E. 1996, AJ, 112, 1487

Johnson, C. I., \& Pilachowski, C. A. 2010, ApJ, 722, 1373

Johnson, C. I., Kraft, R. P., Pilachowski, C. A., et al. 2005, PASP, 117, 1308

Kravtsov, V., Alcaíno, G., Marconi, G., \& Alvarado, F. 2010, A\&A, 512, L6

Kurucz, R. L. 1993, CD-ROM 13, Smithsonian Astrophysical Observatory, Cambridge

Lane, R. R., Brewer, B. J., Kiss, L. L., et al. 2010, ApJ, 711, L122

Lardo, C., Bellazzini, M., Pancino, E., et al. 2011, A\&A, 525, 114

Lee, J.-W., Kang, Y.-W., Lee, J., \& Lee, Y.-W. 2009, Nature, 462, 480
Lind, K., Primas, F., Charbonnel, C., Grundahl, F., \& Asplund, M. 2009, A\&A, 503,545

Lind, K., Charbonnel, C., Decressin, T., et al. 2011, A\&A, 527, A148

Marino, A., Villanova, S., Piotto, G., et al. 2008, A\&A, 490, 625

Marino, A. F., Milone, A. P., Piotto, G., et al. 2011, ApJ, 731, 64

Moro, D., \& Munari, U. 2000, A\&AS, 147, 361

Norris, J., \& Freeman, K. C. 1979, ApJ, 230, L179

Prantzos, N., \& Charbonnel, C. 2006, A\&A, 458, 135

Renzini, A. 2008, MNRAS, 391, 354

Sbordone, L., Salaris, M., Weiss, A., \& Cassisi, S. 2011, A\&A, 534, A9

Schlegel, D. J., Finkbeiner, D. P., \& Davis, M. 1998, ApJ, 500, 525

Skrutskie, M. F. Cutri, R. M., Stiening, R., et al. 2006, AJ, 131, 1163

Sneden, C., Pilachowski, C. A., \& Kraft, R. P. 2000, AJ, 120, 1351

Sneden, C., Kraft, R. P., Guhathakurta, P., Peterson, R. C., \& Fulbright, J. P. 2004, AJ, 127, 2162

Stoughton, C., Lupton, R. H., Bernardi, M., et al. 2002, AJ, 123, 485

Strömgren, B. 1956, Vist. Astron., 2, 1336

Strömgren, B. 1966, ARA\&A, 4, 433

Ventura, P., D’Antona, F., Mazzitelli, I., \& Gratton, R. 2001, ApJ, 550, L65

Yong, D., \& Grundahl, F. 2008, ApJ, 672, L29

Yong, D., Grundahl, F., Johnson, J. A., \& Asplund, M. 2008, ApJ, 684, 1159 